IFT-2004/31

CERN-PH-TH/2004-263

November 2, 2018

\title{
Update on Fermion Mass Models with an Anomalous Horizontal $U(1)$ Symmetry
}

\author{
Piotr H. Chankowski ${ }^{a}$, Kamila Kowalska ${ }^{a}$, Stéphane Lavignac ${ }^{b, 1}$ and \\ Stefan Pokorski ${ }^{a, b}$ \\ ${ }^{a}$ Institute of Theoretical Physics, Warsaw University, Hoża 69, 00-681, Warsaw, Poland \\ ${ }^{b}$ Theory Division, Physics Department, CERN, CH-1211 Geneva 23, Switzerland
}

\begin{abstract}
We reconsider models of fermion masses and mixings based on a gauge anomalous horizontal $U(1)$ symmetry. In the simplest model with a single flavon field and horizontal charges of the same sign for all Standard Model fields, only very few charge assignements are allowed when all experimental data, including neutrino oscillation data, is taken into account. We show that a precise description of the observed fermion masses and mixing angles can easily be obtained by generating sets of the order one parameters left unconstrained by the $U(1)$ symmetry. The corresponding Yukawa matrices show several interesting features which may be important for flavour changing neutral currents and CP violation effects in supersymmetric models.
\end{abstract}

\footnotetext{
${ }^{1}$ Permanent address: Service de Physique Théorique, CEA-Saclay, F-91191 Gif-sur-Yvette Cedex, France.
} 


\section{Introduction}

The origin of the fermion mass and mixing textures is a challenge for physics beyond the Standard Model. One promising approach to that problem is based on hypothetical horizontal symmetries which are spontaneously broken by vacuum expectation values of some "flavon" fields $\Phi$. Hierarchical patterns in the fermion mass matrices can then be explained by the Froggatt-Nielsen mechanism [1, as due to suppression factors $(\langle\Phi\rangle / M)^{n}$, where $M$ is the scale of integrated out physics and the power $n$ depends on the horizontal group charges of the fermion, Higgs and flavon fields.

Fermion mass models based on abelian [2]-[10] and non-abelian [1] horizontal symmetries have been widely discusssed in the literature. Each approach has its own virtues and shortcomings, particularly when realized in supersymmetric models, which we consider here. $U(2)$ and $S U(3)$ symmetries are quite predictive for fermion mass matrices once the pattern of symmetry breaking is specified. However, the quantitative description of fermion masses and mixings requires a rather complicated structure of symmetry breaking. The main shortcoming of $U(1)$ horizontal symmetries as models of fermion masses is the dependence of the quantitative predictions on arbitrary order one coefficients, the remnant of the integrated out unknown physics. In principle also the arbitrariness in the choice of the abelian charges for fermions looks less appealing than the rigid structures of the essentially unique choices of $U(2)$ and $S U(3)$ as continuous horizontal non-abelian symmetry groups. But that last point is balanced by the fact that the breaking of the $U(1)$ symmetry is much simpler.

$U(1)$ gauge group factors are generic in string models. One particularly simple and attractive horizontal symmetry is the anomalous $U(1)$ often found in heterotic string compactifications. Anomaly cancellation by the Green-Schwarz mechanism [12 is possible only under certain conditions which strongly constrain the possible choices of horizontal $U(1)$ charges for fermions. It has already been noticed long ago that those constraints are at a qualitative level amazingly consistent with the quark masses and mixings [4]-9. A particularly appealing feature of the anomalous $U(1)$ is that the symmetry breaking parameter $\langle\Phi\rangle / M$ can be computed in terms of the horizontal charges, and that its value in explicit models turns out to be very close to the Cabibbo angle. Furthermore, with positive charges for matter fields and negative for the flavon field (or vice-versa), this vacuum is unique.

The purpose of this note is to update the predictions for fermion masses of the simplest, string-inspired gauge anomalous $U(1)$ models with a single flavon field $\Phi$. We are motivated by several factors. One is the recent experimental progress in neutrino physics. Neutrino masses and mixings can be well described by models based on horizontal $U(1)$ symmetries, and it is therefore interesting to check what phenomenological constraints, in addition to the GreenSchwarz anomaly cancellation conditions, are put on the horizontal charges by the requirement that the same gauged $U(1)$ symmetry explains both the quark and lepton mass hierarchies. In fact, assuming that all Standard Model fields have horizontal charges of the same sign, we find a very limited number of possible charge assignments.

Furthermore, it is interesting to check the success of these models beyond the qualitative 
level. In this paper, we perform a complete numerical fit of a few representative models to fermion masses and mixings, with the inclusion of complex order one coefficients and with attention paid to the $\tan \beta$ dependence.

Finally, in supersymmetric models there are new sources of flavour and CP violation induced by virtual sfermion exchanges. Sfermion mass matrices depend both on the pattern of supersymmetry breaking and on the rotations to the super-CKM basis which are determined by the fermion mass matrices. Thus, it is useful to have an explicit set of successful fermion mass models, defined by a set of horizontal charges and complex order one coefficients. The fact that only a small number of charge assignments are allowed makes it possible to test the phenomenological implications of an anomalous gauge horizontal symmetry. In a forthcoming paper, we shall use this set of models to study flavour changing neutral currents (FCNCs) in supersymmetry breaking scenarios with dominant $D$-term breaking.

The paper is organized as follows. In the next section, we review the basic properties of fermion mass models based on an anomalous $U(1)$ and list the phenomenologically allowed charge assignements. In section 3 we generate sets of complex order one coefficients giving a precise description of fermion masses and mixings, and study the features of the associated Yukawa matrices relevant for FCNC and CP violating processes. Finally, we present our conclusions in section 4 .

\section{Constraints on $U(1)_{X}$ charges}

The models we consider in this paper are extensions of the $(R$-parity conserving) MSSM with a horizontal gauge abelian symmetry $U(1)_{X}$ and a chiral superfield $\hat{\Phi}$ with $X$-charge normalized to -1 , whose vacuum expectation value breaks the horizontal symmetry. The $X$-charges of the MSSM superfields $\hat{Q}_{A}, \hat{U}_{A}^{c}, \hat{D}_{A}^{c}, \hat{L}_{A}, \hat{E}_{A}^{c}(A=1,2,3), \hat{H}_{u}$ and $\hat{H}_{d}$ are denoted by $q_{A}$, $\bar{u}_{A}, \bar{d}_{A}, l_{A}, \bar{e}_{A}, h_{u}$ and $h_{d}$, respectively. The model also contains three right-handed neutrino superfields $\hat{N}_{A}^{c}$ with charges $\bar{n}_{A}$, which are needed to generate the neutrino masses via the seesaw mechanism [13. No additional matter charged under the SM gauge group is assumed, although the forthcoming analysis would not be altered by the presence of vector-like matter under both the SM gauge group and $U(1)_{X}$. The Yukawa couplings of quarks and leptons are generated via the Froggatt-Nielsen mechanism [1], from nonrenormalizable superpotential terms of the form:

$$
C_{u}^{A B} \hat{U}_{A}^{c} \hat{Q}_{B} \hat{H}_{u}\left(\frac{\hat{\Phi}}{M}\right)^{\bar{u}_{A}+q_{B}+h_{u}}
$$

These terms arise upon integrating out heavy vector-like (super)fields, the so-called FroggattNielsen fields, whose characteristic mass scale is $M$. In string compactifications, the rôle of the Froggatt-Nielsen fields is played by massive string modes, and $M$ is identified with the Planck scale (although vector-like fields may also be present among the massless string modes). After breaking of the horizontal symmetry by the VEV of the scalar component $\phi$ of the superfield $\hat{\Phi}$, 
one obtains effective Yukawa couplings suppressed by powers of the small parameter $\epsilon \equiv\langle\phi\rangle / M$ :

$$
\mathbf{Y}_{u}^{A B}=C_{u}^{A B} \epsilon^{\bar{u}_{A}+q_{B}+h_{u}}
$$

The factors $C_{u}^{A B}$ are not constrained by the horizontal symmetry and are assumed to be of order one. Then the hierarchy of Yukawa couplings is determined, up to these unknown factors, by the charges of the MSSM fields. Since holomorphicity of the superpotential forbids nonrenormalizable terms with a negative power of the superfield $\hat{\Phi}$, one has $\mathbf{Y}_{u}^{A B}=0$ if ${ }^{2}$ $\bar{u}_{A}+q_{B}+h_{u}<0$. Similarly, $\mathbf{Y}_{u}^{A B}=0$ if $\bar{u}_{A}+q_{B}+h_{u}$ is not an integer.

Within the above assumptions, one can show that the horizontal abelian symmetry has to be anomalous [9] (see also Refs. [4, 5]). Indeed, one has the following relations:

$$
\begin{aligned}
& \operatorname{det} M_{u} \operatorname{det} M_{d} \sim v_{u}^{3} v_{d}^{3} \epsilon^{C_{3}+3\left(h_{u}+h_{d}\right)}, \\
& \operatorname{det} M_{d} / \operatorname{det} M_{e} \sim \epsilon^{-\frac{1}{2}\left(C_{1}+C_{2}-\frac{8}{3} C_{3}\right)+h_{u}+h_{d}},
\end{aligned}
$$

where $C_{3} \equiv \sum_{A}\left(2 q_{A}+\bar{u}_{A}+\bar{d}_{A}\right), C_{2} \equiv \sum_{A}\left(3 q_{A}+l_{A}\right)+h_{u}+h_{d}$ and $C_{1} \equiv \frac{1}{3} \sum_{A}\left(q_{A}+8 \bar{u}_{A}+2 \bar{d}_{A}+\right.$ $\left.3 l_{A}+6 \bar{e}_{A}\right)+h_{u}+h_{d}$ are the coefficients of the mixed $S U(3)_{C^{-}} S U(3)_{c^{-}} U(1)_{X}, S U(2)_{L^{-}} S U(2)_{L^{-}}$ $U(1)_{X}$ and $U(1)_{Y^{-}} U(1)_{Y^{-}} U(1)_{X}$ anomalies, respectively, and $v_{u, d}$ are the vacuum expectation values of the two Higgs doublets. If all anomaly coefficients were vanishing, the hierarchy among quark masses would require a large, positive value of $h_{u}+h_{d}\left(h_{u}+h_{d}=6-8\right.$ for $\epsilon=\lambda$, where $\lambda$ is the sine of the Cabibbo angle, depending on the value of $\tan \beta$ ), while the relation (44) would require a much smaller value $\left(h_{u}+h_{d}=1\right.$ or 2 for $\left.\epsilon=\lambda\right)$. Therefore, the horizontal symmetry has to be anomalous if it is to explain the observed fermion mass hierarchy.

This fact provides the main motivation for considering a gauged horizontal symmetry. It is well-known that abelian gauge anomalies can be compensated for by the Green-Schwarz mechanism [12], as is common in four-dimensional heterotic string compactifications ${ }^{3}$. This requires that the following relations between anomaly coefficients be satisfied:

$$
\frac{C_{1}}{k_{1}}=\frac{C_{2}}{k_{2}}=\frac{C_{3}}{k_{3}}=\frac{C_{X}}{k_{X}}=\frac{\operatorname{Tr} X}{12}, \quad C_{X X Y}=0,
$$

where $C_{X}$ is the coefficient of the cubic $U(1)_{X}$ anomaly, $\operatorname{Tr} X$ is the coefficient of the mixed $U(1)_{X}$-gravitational anomaly, and $k_{a}$ is the Kac-Moody level of the gauge group $G_{a}$, which

\footnotetext{
${ }^{2}$ This conclusion can be evaded if the Froggatt-Nielsen fields, instead of being vector-like under both the Standard Model gauge group and the $U(1)_{X}$ group as usually assumed, are chiral under $U(1)_{X}$. In such a case effective operators carrying a negative $X$-charge can be induced in the low-energy effective theory. Moreover, even if the Froggatt-Nielsen fields are vector-like under $U(1)_{X}$, effective operators $\hat{L}_{A} \hat{L}_{B} \hat{H}_{u} \hat{H}_{u}$ with a negative $X$-charge may be induced by the seesaw mechanism, since right-handed neutrino superfields are chiral under $U(1)_{X}$ (see e.g. the model of Ref. [14).

${ }^{3}$ Anomalous $U(1)$ 's are also common in four-dimensional open string compactifications. However, while heterotic string compactifications contain at most one anomalous abelian gauge group factor, open string compactifications may contain several anomalous $U(1)$ 's whose anomalies are compensated for by a generalized Green-Schwarz mechanism [15]. In this case, the Green-Schwarz anomaly conditions are less constraining than in the heterotic case, and the scale of breaking of the anomalous $U(1)$ 's depends on twisted moduli vevs, which are fixed by unknown nonperturbative physics. For these reasons, we prefer to consider the case of an anomalous $U(1)$ of the heterotic type, with its anomalies canceled by the universal Green-Schwarz mechanism.
} 
depends on the compactification. The Kac-Moody level of a non-abelian gauge group is an integer, while the Kac-Moody level of an abelian gauge group can be fractional. In the following, we shall assume $k_{2}=k_{3}$, as is the case in most of (if not all of) the heterotic string compactifications constructed so far. Since the $U(1)_{X^{-}} U(1)_{X^{-}} U(1)_{Y}$ anomaly cannot be compensated for by the Green-Schwarz mechanism, its coefficient $C_{X X Y}$ has to vanish by itself.

A nice feature of the Green-Schwarz mechanism is that the value of the Weinberg angle at the string scale is determined by the ratio of the anomaly coefficients $C_{1}$ and $C_{2}$ [16]. Indeed, upon combining the anomaly conditions with the gauge coupling unification relation $k_{1} g_{1}^{2}=k_{2} g_{2}^{2}=\cdots=g_{\text {string }}^{2}$ valid at the string scale, one obtains:

$$
\sin \theta_{W}^{2}\left(M_{\text {string }}\right)=\frac{C_{2}}{C_{1}+C_{2}}
$$

The canonical value of the Weinberg angle at the GUT scale $\left(\sin ^{2} \theta_{W}=3 / 8\right)$ is obtained for $C_{1} / C_{2}=5 / 3$, or equivalently for the normalization $k_{1} / k_{2}=5 / 3$, which is well known from string model builders. As observed in Refs. [4, 5, 8], this normalization (together with $k_{2}=k_{3}$ ) leads through Eq. (4) to $\frac{m_{d} m_{s} m_{b}}{m_{e} m_{\mu} m_{\tau}} \sim \epsilon^{h_{u}+h_{d}}$, which given the uncertainty due to the order one coefficients is consistent with data ${ }^{4}$ for $h_{u}+h_{d}=0, \pm 1$ or even \pm 2 . Such values of $h_{u}+h_{d}$ are suitable for electroweak symmetry breaking if the $\mu$-term is generated from the Giudice-Masiero mechanism [17]. In the following, we shall fix the Weinberg angle at its canonical GUT value and impose $C_{1} / C_{2}=5 / 3$.

Another nice feature of an anomalous $U(1)_{X}$ symmetry is that its breaking scale, hence the small expansion parameter $\epsilon$, is determined by the value of the gauge coupling at the unification scale and by the anomaly coefficient $C_{2}$ [14]. Indeed, due to the fact that $\operatorname{Tr} X \neq 0$, a Fayet-Iliopoulos term is generated at the one-loop level [18, 19]:

$$
\xi^{2}=\frac{g_{\text {string }}^{2}}{192 \pi^{2}} \operatorname{Tr}\left(X M^{2}\right),
$$

where $g_{\text {string }}$ is the string coupling and $M$ the reduced Planck mass. Provided that $X_{\Phi} \operatorname{Tr} X<0$, this triggers a nonzero VEV of the scalar component $\phi$ of the superfield $\hat{\Phi}$, which sets the value of the $\epsilon$ parameter to [14]:

$$
\epsilon=\sqrt{\frac{g_{\text {string }}^{2}}{192 \pi^{2}} \operatorname{Tr} X}=\sqrt{\frac{\alpha_{2}}{4 \pi} C_{2}},
$$

where we have used the relations $k_{2} g_{2}^{2}=g_{\text {string }}^{2}$ and $C_{2} / k_{2}=\operatorname{Tr} X / 12$ (recall that $X_{\Phi}=-1$ ). As we shall see later, in realistic models the value of $\epsilon$ is very close to the Cabibbo angle, $\lambda \simeq 0.22$.

We now look for solutions to the anomaly constraints. In addition, we require the absence of kinetic mixing between the anomalous $U(1)$ and the hypercharge, i.e. $\operatorname{Tr}(X Y)=0$. As noticed in Ref. [20, this condition also prevents the generation of a large one-loop Fayet-Iliopoulos term for the hypercharge [21] in the scenario in which the dominant contribution to soft scalar

\footnotetext{
${ }^{4}$ Assuming approximate bottom-tau Yukawa coupling unification at the GUT scale, this mass ratio is between 0.75 and 0.9 for the central values of the fermion masses in the $\tan \beta$ range $5-60$, which favours $h_{u}+h_{d}=0$.
} 
masses comes from the anomalous $D$-term. Such a Fayet-Iliopoulos term could have induced charge and colour breaking minima. We look for models with the $X$-charges of all quark, lepton and Higgs superfields positive or zero, which is sufficient to ensure the uniqueness of the vacuum that breaks the anomalous $U(1)_{X}$ (see Ref. 22] for a detailed discussion of flat directions).

The relevant anomaly coefficients read:

$$
\begin{aligned}
C_{1} & =\frac{1}{3} \sum_{A}\left(q_{A}+8 \bar{u}_{A}+2 \bar{d}_{A}+3 l_{A}+6 \bar{e}_{A}\right)+h_{u}+h_{d}, \\
C_{2} & =\sum_{A}\left(3 q_{A}+l_{A}\right)+h_{u}+h_{d}, \\
C_{3} & =\sum_{A}\left(2 q_{A}+\bar{u}_{A}+\bar{d}_{A}\right), \\
C_{X X Y} & =\sum_{A}\left(q_{A}^{2}-2 \bar{u}_{A}^{2}+\bar{d}_{A}^{2}-l_{A}^{2}+\bar{e}_{A}^{2}\right)+h_{u}^{2}-h_{d}^{2}, \\
C_{X} & =\frac{2}{3} \sum_{A}\left(6 q_{A}^{3}+3 \bar{u}_{A}^{3}+3 \bar{d}_{A}^{3}+2 l_{A}^{3}+\bar{e}_{A}^{3}+n_{A}^{3}\right)+h_{u}^{3}+h_{d}^{3}-1+C_{X}^{\prime}, \\
\operatorname{Tr} X & =\sum_{A}\left(6 q_{A}+3 \bar{u}_{A}+3 \bar{d}_{A}+2 l_{A}+\bar{e}_{A}+\bar{n}_{A}\right)+2\left(h_{u}+h_{d}\right)-1+C_{g}^{\prime},
\end{aligned}
$$

where $C_{X}^{\prime}$ and $C_{g}^{\prime}$ stand for the contributions of additional SM singlets or vector-like representations charged under $U(1)_{X}$ (which may be charged under some hidden gauge group) to the cubic $U(1)_{X}$ anomaly and to the mixed gravitational anomaly, respectively. Finally, $\operatorname{Tr}(X Y)$ reads:

$$
\operatorname{Tr}(X Y)=2 \sum_{A}\left(q_{A}-2 \bar{u}_{A}+\bar{d}_{A}-l_{A}+\bar{e}_{A}\right)+2\left(h_{u}-h_{d}\right) .
$$

The constraints ${ }^{5}$ :

$$
C_{2}=C_{3}, \quad C_{2}=\frac{3}{5} C_{1}, \quad C_{X X Y}=0 \quad \text { and } \quad \operatorname{Tr}(X Y)=0
$$

can be rewritten as:

$$
\begin{aligned}
& \sum_{A}\left(\bar{u}_{A}-q_{A}\right)-\frac{1}{2} h_{u}=0, \\
& \sum_{A}\left(\bar{d}_{A}-l_{A}\right)-\frac{1}{2} h_{u}-h_{d}=0, \\
& \sum_{A}\left(\bar{u}_{A}-\bar{e}_{A}\right)-h_{u}=0, \\
& \sum_{A}\left(q_{A}^{2}-2 \bar{u}_{A}^{2}+\bar{d}_{A}^{2}-l_{A}^{2}+\bar{e}_{A}^{2}\right)+h_{u}^{2}-h_{d}^{2}=0 .
\end{aligned}
$$

\footnotetext{
${ }^{5}$ We assume that $C_{X}^{\prime}, C_{g}^{\prime}$ and the Kac-Moody level $k_{X}$ are such that the Green-Schwarz conditions are satisfied for the cubic $U(1)_{X}$ anomaly and for the mixed gravitational anomaly.
} 
We look for solutions to Eqs. (17)-(20) which successfully reproduce the observed quark and lepton mass and mixing hierarchies with all matter charges non-negative. Since the Yukawa couplings are generated at the scale of breaking of the anomalous $U(1)_{X}$ (which for definiteness we identify with the scale at which gauge couplings unify in the MSSM, $M_{G U T} \simeq 2 \times 10^{16}$ $\mathrm{GeV}$ ), we must consider the masses and mixings renormalized also at this scale. In the quark sector, the renormalization of the CKM matrix affects, to a very good approximation, only the parameter $A$ of the Wolfenstein parametrization, while $\lambda, \rho$ and $\eta$ almost do not evolve with energy [23, 24, 25, 26]. The scale dependence of the quark mass ratios $m_{d} / m_{s}$ and $m_{u} / m_{c}$ is also very weak. Numerically for $\tan \beta$ values in the range $3.5-50$ we find:

$$
\frac{m_{s}(\mu)}{m_{b}(\mu)}=\chi(\mu) \frac{m_{s}\left(M_{Z}\right)}{m_{b}\left(M_{Z}\right)}, \quad \frac{m_{c}(\mu)}{m_{t}(\mu)}=\chi^{3}(\mu) \frac{m_{c}\left(M_{Z}\right)}{m_{t}\left(M_{Z}\right)}
$$

with $\chi\left(M_{G U T}\right) \simeq(0.75-0.9)$. This leads to the following approximate hierarchies of quark mixing angles and mass ratios close to the GUT scale, expressed in powers of the Cabibbo angle $\lambda \simeq 0.22$ :

$$
\begin{gathered}
\left|V_{u s}\right| \simeq\left|V_{c d}\right| \simeq \lambda, \quad\left|V_{u b}\right| \sim \lambda^{4}, \quad\left|V_{c b}\right| \sim \lambda^{2}, \quad\left|V_{t d}\right| \sim \lambda^{4}-\lambda^{3}, \quad\left|V_{t s}\right| \sim \lambda^{2}, \\
\frac{m_{d}}{m_{s}} \sim \lambda^{2}, \quad \frac{m_{s}}{m_{b}} \sim \frac{1}{2} \lambda^{2}, \quad \frac{m_{u}}{m_{c}} \sim \lambda^{4}, \quad \frac{m_{c}}{m_{t}} \sim \lambda^{4} .
\end{gathered}
$$

For light quarks we have used here the central values of the PDG estimates [27]. The dependence on $\tan \beta$ of the GUT scale values of these mass ratios and CKM elements is weak in the considered range of $\tan \beta$ values.

In the lepton sector, the charged lepton mass hierarchy is:

$$
\frac{m_{e}}{m_{\mu}} \sim \frac{1}{2} \lambda^{3}, \quad \frac{m_{\mu}}{m_{\tau}} \sim \lambda^{2} .
$$

If one assumes a hierarchical neutrino mass spectrum, i.e. $m_{\nu_{3}} \approx \sqrt{\Delta m_{\mathrm{atm}}^{2}}$ and $m_{\nu_{2}} \approx \sqrt{\Delta m_{\mathrm{sol}}^{2}}$, the scale dependence of lepton mixing angles and neutrino masses is very weak [28, 26], and one can take, at the scale where right-handed neutrinos decouple:

$$
\left|U_{\alpha A}\right| \sim 1, \quad(\alpha, A) \neq(e, 3), \quad\left|U_{e 3}\right|<\lambda, \quad \frac{m_{\nu_{2}}}{m_{\nu_{3}}} \sim \lambda, \quad \frac{m_{\nu_{1}}}{m_{\nu_{2}}}<1 .
$$

Eqs. (17) to (20), together with the assumption of positive $X$-charges for the MSSM superfields, are very restrictive and only a limited number of charge assignments compatible with these constraints give a satisfactory account of the observed mass and mixing hierarchies. Let us first consider the quark sector. The large top quark Yukawa coupling is accounted for by choosing $q_{3}=\bar{u}_{3}=h_{u}=0$. Furthermore, the quark mass ratios and mixing angles are given by the simple expressions:

$$
\begin{gathered}
\frac{m_{u_{A}}}{m_{u_{B}}} \sim \epsilon^{\left(q_{A}-q_{B}\right)+\left(\bar{u}_{A}-\bar{u}_{B}\right)}, \quad \frac{m_{d_{A}}}{m_{d_{B}}} \sim \epsilon^{\left(q_{A}-q_{B}\right)+\left(\bar{d}_{A}-\bar{d}_{B}\right)}, \quad \frac{m_{t}}{m_{b}} \sim \tan \beta \epsilon^{-\left(\bar{d}_{3}+h_{d}\right)}, \\
V_{A B} \sim \epsilon^{\left|q_{A}-q_{B}\right|} .
\end{gathered}
$$


The symbol $\sim$ reminds us that the above relations contain unknown factors of order one, so that the actual values of the mass ratios and mixing angles may slightly depart from the naive "power counting". With this remark in mind, and assuming $\epsilon \simeq \lambda$, we find that the CKM matrix is correctly reproduced by $q_{A}=(3,2,0), q_{A}=(4,3,0)$ or by $q_{A}=(4,2,0)$. In each case, the naive "power counting" disagrees by at most one power of $\lambda$ with the measured value of one or two CKM angles: $\left|V_{u b}\right|$ for $q_{A}=(3,2,0),\left|V_{c b}\right|$ and $\left|V_{t s}\right|$ for $q_{A}=(4,3,0),\left|V_{u s}\right|$ and $\left|V_{c d}\right|$ for $q_{A}=(4,2,0)$. In the lepton sector, the experimental data on neutrino oscillations strongly constrain the $l_{A}$ (for a recent review, see e.g. Ref. [29]). In the absence of holomorphic zeroes in the Dirac and Majorana mass matrices, the seesaw mechanism yields an effective light neutrino mass matrix of the form:

$$
\mathbf{M}_{\nu} \sim \frac{v_{u}^{2} \epsilon^{2\left(l_{3}+h_{u}\right)}}{M_{R}}\left(\begin{array}{ccc}
\epsilon^{2\left(l_{1}-l_{3}\right)} & \epsilon^{\left(l_{1}-l_{3}\right)+\left(l_{2}-l_{3}\right)} & \epsilon^{\left(l_{1}-l_{3}\right)} \\
\epsilon^{\left(l_{1}-l_{3}\right)+\left(l_{2}-l_{3}\right)} & \epsilon^{2\left(l_{2}-l_{3}\right)} & \epsilon^{\left(l_{2}-l_{3}\right)} \\
\epsilon^{\left(l_{1}-l_{3}\right)} & \epsilon^{\left(l_{2}-l_{3}\right)} & 1
\end{array}\right)
$$

where $M_{R}$ is the scale of right-handed neutrino masses. Such a mass matrix can easily reproduce the hierarchical neutrino mass spectrum. In order to reproduce both the large atmospheric mixing angle and the hierarchy between the atmospheric and solar mass scales, one must choose $l_{2}=l_{3}$ and allow for a mild tuning between the order one entries in the lower right $2 \times 2$ submatrix of $\mathbf{M}_{\nu}$, of order $\sqrt{\Delta m_{\text {sol }}^{2} / \Delta m_{\text {atm }}^{2}} \approx 0.2$. Due to this tuning, the solar mixing angle $\theta_{12}$ comes out "large" provided that $l_{1}-l_{3}=1$ or 2 (see Appendix B for details). This in turn implies that $\left|U_{e 3}\right| \sim \epsilon^{l_{1}-l_{3}}$ should be rather large, and even close to its present experimental limit in the case $l_{1}-l_{3}=1$.

For a given choice of the $q_{A}$ and the $l_{A}$ dictated by the CKM and PMNS mixing matrices, the remaining $X$-charges of the model are constrained both by the quark and charged lepton masses and by Eqs. (17) to (20). We list below the solutions for the $X$-charge assignments satisfying the constraints (17) to (20) for which the predictions obtained from naive power counting are in reasonably good agreement with experimental data on fermion masses and mixings, when extrapolated to the GUT scale:

$$
\begin{array}{ll}
\mathbf{1 :} & q_{A}=\bar{u}_{A}=\bar{e}_{A}=(3,2,0), \quad \bar{d}_{A}=l_{A}=\left(l_{1}, l_{3}, l_{3}\right), \quad h_{u}=h_{d}=0, \\
& {\left[l_{1}-l_{3}=1 \text { or } 2 ; l_{3}=0,1,2 \text { or } 3\right]} \\
\mathbf{2 :} & q_{A}=\bar{u}_{A}=\bar{e}_{A}=(3,2,0), \quad \bar{d}_{A}=(2,1,0), \quad l_{A}=(1,0,0), \quad h_{u}=0, \quad h_{d}=2, \\
\mathbf{3 :} & q_{A}=\bar{u}_{A}=\bar{e}_{A}=(3,2,0), \quad \bar{d}_{A}=(1,1,0), \quad l_{A}=(1,0,0), \quad h_{u}=0, \quad h_{d}=1, \\
\mathbf{4 :} & q_{A}=\bar{u}_{A}=\bar{e}_{A}=(3,2,0), \quad \bar{d}_{A}=(2,1,0), l_{A}=(2,0,0), \quad h_{u}=0, \quad h_{d}=1, \\
\mathbf{5 :} & q_{A}=\bar{u}_{A}=e_{A}=(4,2,0), \quad \bar{d}_{A}=l_{A}=\left(l_{3}+1, l_{3}, l_{3}\right), \quad h_{u}=h_{d}=0, \\
& {\left[l_{3}=0,1,2 \text { or } 3\right]} \\
\mathbf{6 :} & q_{A}=\bar{u}_{A}=\bar{e}_{A}=(4,2,0), \quad \bar{d}_{A}=(1,1,0), \quad l_{A}=(1,0,0), \quad h_{u}=0, \quad h_{d}=1 .
\end{array}
$$

Several of these solutions can be found in the literature. The constraints (17) to (20) are automatically satisfied if $h_{u}=h_{d}=0$ and the horizontal symmetry commutes with $S U(5)$, as already noticed in Ref. [20]. This is the case for solutions $\mathbf{1}$ and $\mathbf{5}$. We were not able to find solutions with $h_{u}=h_{d}=0$ that are not compatible with the $S U(5)$ symmetry. In all solutions, 
the naive predictions slightly depart from the observed values for some quantities, and one has to rely on the effect of the unconstrained order one parameters to correct them. In particular, solutions 1 to 4 naively predict $m_{u} / m_{c} \sim \lambda^{2}$ and $\left|V_{u b}\right| \sim \lambda^{3}$, while solutions $\mathbf{5}$ and $\mathbf{6}$ predict $\left|V_{u s}\right| \sim\left|V_{c d}\right| \sim \lambda^{2}$, but give the correct $m_{u} / m_{c}$ ratio. In addition, solution 1 with $l_{1}-l_{3}=1$ and solutions 2, 3 yield $m_{e} / m_{\mu} \sim \lambda^{2}$; solution 1 with $l_{1}-l_{3}=2$ and solution 5 yield $m_{d} / m_{s} \sim \lambda^{3}$, and solution 3 yields $m_{d} / m_{s} \sim \lambda$. Finally, $l_{1}-l_{3}=1$ is preferred over $l_{1}-l_{3}=2$ by the solar mixing angle [30].

Solutions 1 and 5 with $l_{3}=2$ and solution 2 predict a low value of $\tan \beta, \tan \beta \lesssim 15$; solutions 1 and $\mathbf{5}$ with $l_{3}=1$ and solutions $\mathbf{3}, \mathbf{4}$ and $\mathbf{6}$ require $\tan \beta \sim(15-50)$; and solutions 1 and 5 with $l_{3}=0$ require $\tan \beta \gtrsim 50$. The range of $\tan \beta$ values compatible with a given solution is rather broad due to the effect of the order one coefficients.

Using Eq. (8), one can compute the predicted value of the expansion parameter $\epsilon$. Assuming $\alpha_{2}\left(M_{\text {string }}\right)=\alpha_{U}=\frac{1}{24}$, one obtains (the quoted values scale as $\left.\left(\frac{\alpha_{U}}{1 / 24}\right)^{1 / 2}\right)$ :

$$
\begin{array}{cl}
\epsilon=0.23,0.25,0.27 & \text { for solution } 1 \text { with } l_{1}-l_{3}=1 \text { and } l_{3}=0,1,2, \\
\epsilon=0.24,0.26,0.28 & \text { for solution } 1 \text { with } l_{1}-l_{3}=2 \text { and } l_{3}=0,1,2, \\
\epsilon=0.24 & \text { for solution } 2,3 \text { and } 4, \\
\epsilon=0.25,0.27,0.29 & \text { for solution } 5 \text { with } l_{3}=0,1,2, \\
\epsilon=0.26 & \text { for solution } 6 .
\end{array}
$$

As already stressed in Ref. [14, it is a remarkable success of flavour models based on an anomalous $U(1)$ that the predicted value of $\epsilon$ comes out so close to the Cabibbo angle. Note that the charge assignment $q_{A}=\bar{u}_{A}=\bar{e}_{A}=(2,1,0), l_{A}=\bar{d}_{A}=(1,0,0)+l_{3}$, sometimes considered in the literature, predicts $\epsilon=(0.18-0.23)$, which is too large for this charge assignment to be compatible with the observed fermion masses and mixings.

\section{Precision description of fermion masses and mixings}

The fact that only a small number of horizontal charge assignments are phenomenologically acceptable, together with the interesting theoretical aspects discussed in the previous section, makes the simplest anomalous $U(1)$ models for fermion masses worth further, more quantitative study. Such a study may also be useful for studying various aspects of the supersymmetric flavour problem and CP violation with hierarchical fermion mass matrices.

The charge assignments 1-6 in Eqs. (28)-(33) predict the hierarchical structure of the quark and charged lepton Yukawa couplings up to order one factors $C_{u, d, e}^{A B}$, which should be viewed as fixed by some unknown physics which has been integrated out. The freedom in these factors can be used to obtain a precise description of the fermion masses and mixings ${ }^{6}$; it is the purpose of this section to find such sets of coefficients $C_{u, d, e}^{A B}$ and to discuss their properties.

\footnotetext{
${ }^{6}$ For a quantitative study of the predictions in the neutrino sector, we refer to Ref. 30, which however only considered the choice $l_{1}-l_{3}=1$ ("semi-anarchical" case). We argued, on the basis of the analytical formulae given in appendix $\mathrm{B}$, that the choice $l_{1}-l_{3}=2$ also leads to a satisfactory description of neutrino masses and
} 
Before doing so, let us notice that, among the acceptable $U(1)_{X}$ charge assignments, two (namely charge assignments $\mathbf{1}$ and 5) are consistent with $S U(5) \times U(1)_{X}$ symmetry, and can therefore be reconciled with Grand Unification of elementary forces. Moreover, those are the only acceptable charge assignments for $h_{u}=h_{d}=0$, for which, as mentioned earlier, there is some (although not strong) phenomenological preference. Charge assignments $\mathbf{3}$ and $\mathbf{6}$, although not compatible with $S U(5)$ symmetry, are interesting in the context of the supersymmetric flavour problem because the right-handed down and strange quark superfields have the same horizontal charge, which in some supersymmetry breaking scenarios may suppress the squark contribution to kaon mixing.

If one interprets the fact that the charge assignments $\mathbf{1}$ and $\mathbf{5}$ are compatible with $S U(5) \times$ $U(1)_{X}$ symmetry as the manifestation of an underlying Grand Unified Theory, one should impose the following (GUT-scale) constraints on the order one coefficients: $C_{u}^{A B}=C_{u}^{B A}$ and $C_{d}^{A B}=C_{e}^{B A}$. The second constraint leads to the well-known $S U(5)$ relations $m_{e} / m_{\mu}=m_{d} / m_{s}$ and $m_{\mu} / m_{\tau}=m_{s} / m_{b}$, which are in gross disagreement with the measured fermion masses and must be corrected [31. This can be done through the contribution of renormalizable [31] or non-renormalizable 32 operators to the Yukawa matrices. Following Ref. [33], we shall introduce an additional $U(1)_{X}$ singlet superfield $\hat{\Sigma}$ transforming as a 75 of $S U(5)$, which has non-renormalizable couplings to fermions of the form $\overline{5} \mathbf{1 0} \bar{H} \hat{\Sigma} / M$. The Yukawa couplings of the down-type quarks and charged leptons then arise from the two $S U(5) \times U(1)_{X}$ invariant superpotential terms:

$$
W=\left(\overline{\mathbf{5}}^{A} C_{1}^{A B} \mathbf{1 0}^{B} \bar{H}+\frac{\hat{\Sigma}}{M} \overline{\mathbf{5}}^{A} C_{2}^{A B} \mathbf{1 0}^{B} \bar{H}\right)\left(\frac{\hat{\Phi}}{M}\right)^{\bar{d}_{A}+q_{B}+h_{d}}
$$

which, after the scalar components of $\hat{\Phi}$ and $\hat{\Sigma}$ acquire VEVs, lead to:

$$
\begin{gathered}
\mathbf{Y}_{d}^{A B}=\left(C_{1}^{A B}+\kappa C_{2}^{A B}\right) \epsilon^{\bar{d}_{A}+q_{B}+h_{d}} \\
\mathbf{Y}_{e}^{A B}=\left(C_{1}^{B A}-3 \kappa C_{2}^{B A}\right) \epsilon^{\bar{d}_{B}+q_{A}+h_{d}}
\end{gathered}
$$

where $\kappa \equiv\langle\Sigma\rangle / M$. In our numerical fits, we take $\kappa=0.3$, which makes it easy to account for the difference between down-type quark and charged letpon masses.

In order to test in a quantitative way the ability of the $U(1)_{X}$ symmetry to describe the fermion masses and mixings, we look for sets of 24 complex coefficients $C_{u}^{A B}=C_{u}^{B A}, C_{1}^{A B}$ and $C_{2}^{A B}$ that reproduce the 9 quark and charged lepton masses and the 4 parameters of the CKM matrix. It is also interesting to check how well the various charge assignments reproduce the observed values of the fermion masses and mixings for randomly generated coefficients. This is illustrated in Figs. 1 and 2 for charge assignments 1 (with two different choices for $l_{A}=\bar{d}_{A}$ ), $\mathbf{5}$ and $\mathbf{6}$. For randomly generated sets of coefficients $\left\{C_{u, 1,2}^{A B}\right\}$ with arbitrary phases and moduli in the range $0.3-3$, each histogram shows the relative number of sets of coefficients leading to a given value of the ratio $R(Q) \equiv Q\left(M_{G U T}\right)_{\text {predicted }} / Q\left(M_{G U T}\right)_{\text {evolved }}$ for some particular

mixings. In the following, we shall not discuss the neutrino sector again and shall focus on the charged fermion sector. 

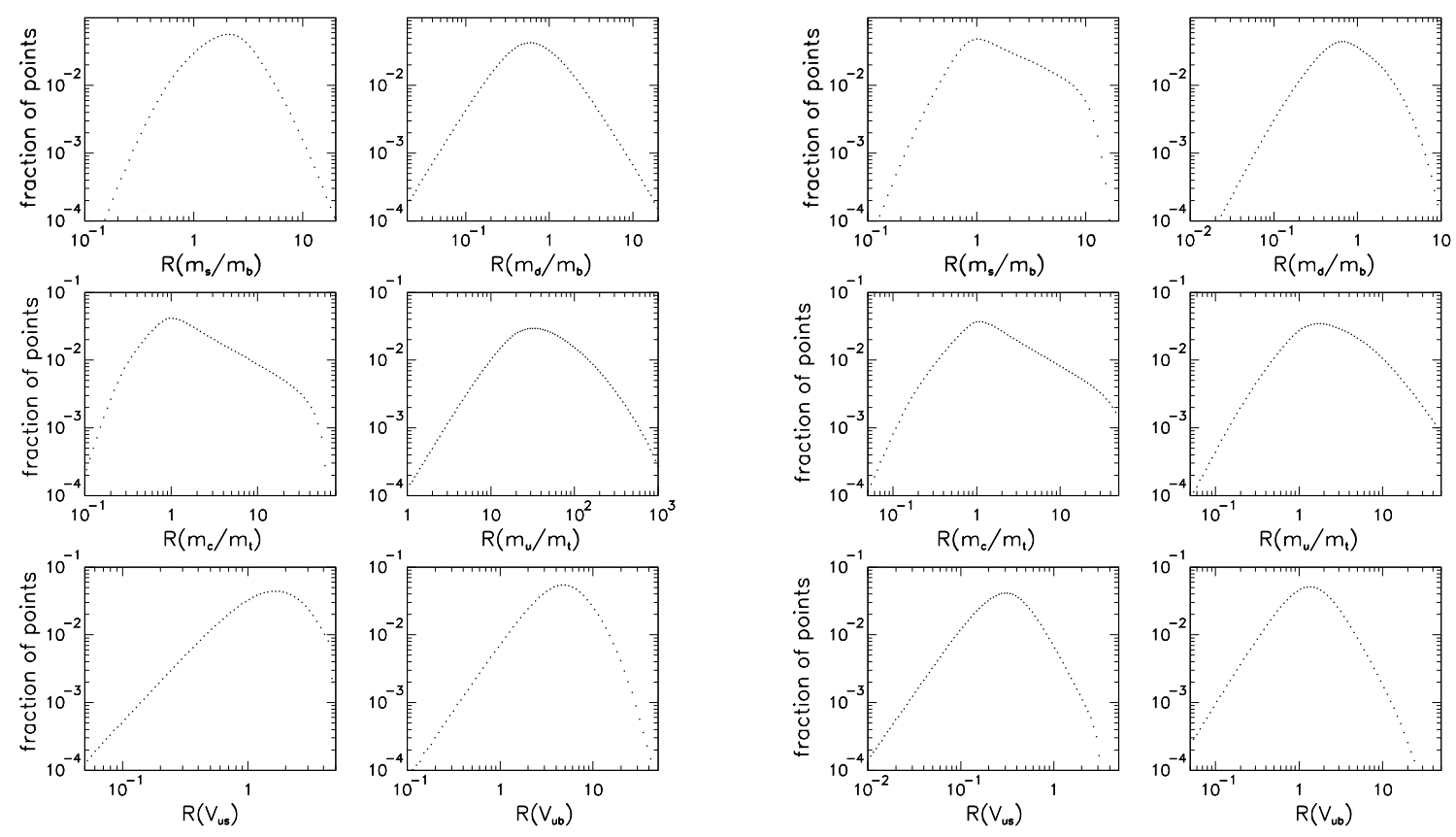

Figure 1: Histograms of $R\left(m_{s} / m_{b}\right), R\left(m_{d} / m_{b}\right), R\left(m_{c} / m_{t}\right), R\left(m_{u} / m_{t}\right), R\left(V_{u s}\right)$ and $R\left(V_{u b}\right)$, where $R(Q) \equiv Q\left(M_{G U T}\right)_{\text {predicted }} / Q\left(M_{G U T}\right)_{\text {evolved }}$, for charge assignment 1 with $l_{A}=\bar{d}_{A}=$ $(4,2,2)$ and $\tan \beta=15$ (left set of panels) and for charge assignment 6 with $\tan \beta=45$ (right set of panels).

mass ratio or mixing angle $Q$. The positions of the peaks are nicely consistent with the naive "power counting" of Eq. (26) and with the qualitative discussion of the previous section. Since all charge assignments $\mathbf{1}-\mathbf{6}$ share similar qualitative features, we choose model $\mathbf{1}$ for a more detailed analysis.

These histograms also show that, as expected on the basis of the qualitative arguments of the previous section, it is easy to find sets of order one coefficients $\left\{C_{u, 1,2}^{A B}\right\}$ giving a precision description of fermion masses and mixings. In fact, since the number of real parameters by far exceeds the number of observables, there are infinitely many different sets of the coefficients $C_{u, 1,2}^{A B}$ that satisfy the physical requirements. Instead of searching for them by random generation, it is much more efficient to use the equations from Appendix A as constraints on the generation procedure.

In the qualitative discussion of the previous section, we have used the values of the observables at $M_{G U T}$, after evolving them with the RGE from their measured low-energy values. For precision fits, this procedure is inconvenient because the proper inclusion of experimental errors in the renormalization group running is somewhat troublesome. Therefore, we proceed top-down. For a given set of complex coefficients chosen with arbitrary phases and with their moduli in the range $0.3-3$, we calculate the CKM matrix entries and the eigenvalues of the Yukawa matrices at the GUT scale and evolve them with the renormalization group equations 

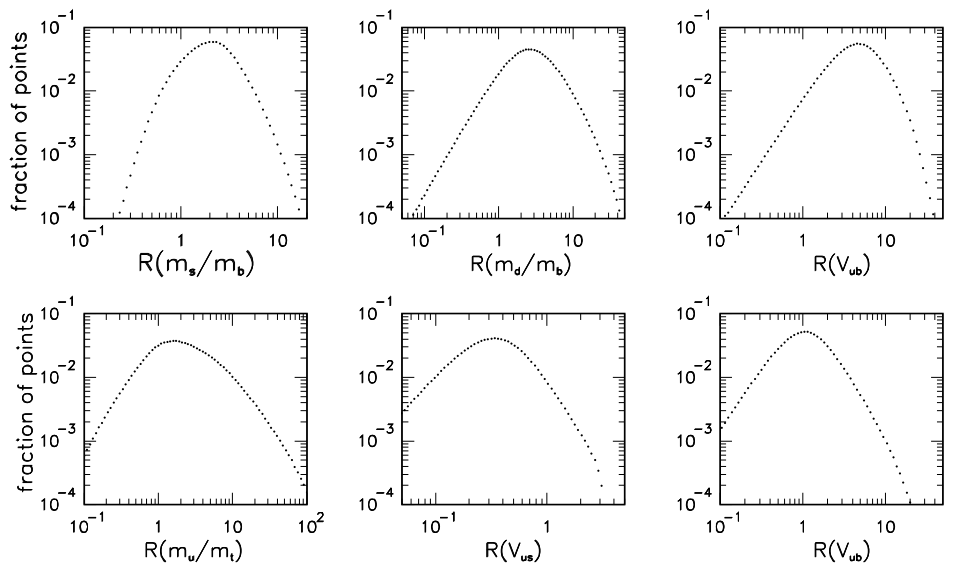

Figure 2: Histograms of $R\left(m_{s} / m_{b}\right), R\left(m_{d} / m_{b}\right)$ and $R\left(V_{u b}\right)$ for charge assignment $\mathbf{1}$ with $l_{A}=$ $\bar{d}_{A}=(3,2,2)$ and $\tan \beta=15$ (upper set of panels), and histograms of $R\left(m_{u} / m_{t}\right), R\left(V_{u s}\right)$ and $R\left(V_{u b}\right)$ for charge assignment 5 with $\tan \beta=15$ (lower set of panels).

down to the scale $M_{Z}\left[24,25\right.$, 26]. The comparison with experimental data is then done at $M_{Z}$, and for a set of coefficients to be acceptable, we require that the predicted values of the CKM elements fall in the following ranges:

$$
\begin{aligned}
0.217<\left|\mathbf{V}_{u s}\right| & <0.231, \\
0.035<\left|\mathbf{V}_{c b}\right| & <0.048, \\
0.065<\left|\frac{\mathbf{V}_{u b}}{\mathbf{V}_{c b}}\right| & <0.105, \\
-73^{0}<\arg \left(\mathbf{V}_{u b}\right) & <-53^{\circ} .
\end{aligned}
$$

The range of "experimental" values of the eigenvalues of the Yukawa matrices at $M_{Z}$ is obtained by starting with the following set of mass values:

$$
\begin{array}{lc}
m_{u}(2 \mathrm{GeV})=(2.75 \pm 2.50) \mathrm{MeV}, & m_{d}(2 \mathrm{GeV})=(6 \pm 4) \mathrm{MeV}, \\
m_{c}(2 \mathrm{GeV})=(1.25 \pm 0.20) \mathrm{GeV}, & m_{s}(2 \mathrm{GeV})=(105 \pm 50) \mathrm{MeV}, \\
m_{b}\left(m_{b}\right)=(4.25 \pm 0.30) \mathrm{GeV}, & m_{t}=(178 \pm 10) \mathrm{GeV}, \\
m_{e}=(0.511 \pm 0.025) \mathrm{MeV}, \quad m_{\mu}=(105.6 \pm 0.5) \mathrm{MeV}, \quad m_{\tau}=(1.778 \pm 0.1) \mathrm{GeV} .
\end{array}
$$

For quark masses, these ranges are twice as broad as the PDG estimates. For lepton masses we assigned arbitrary uncertainties, since the actual experimental errors are much smaller than the uncertainty in the RG evolution due to e.g. the errors on the gauge couplings or quark Yukawa couplings, or to the fact that we are only using two-loop RG equations. The Yukawa couplings at the $M_{Z}$ scale are obtained by evolving these masses up using the 2-loop QCD RG equations (and taking into account the uncertainty in the strong coupling: $\alpha_{s}\left(M_{Z}\right)=0.1185 \pm 0.005$ ). The top quark Yukawa at the $M_{Z}$ scale is adjusted iteratively so that its RG trajectory passes through the right value at the $m_{t}$ scale. In this way we establish the allowed ranges of the Yukawa couplings at the $M_{Z}$ scale. 

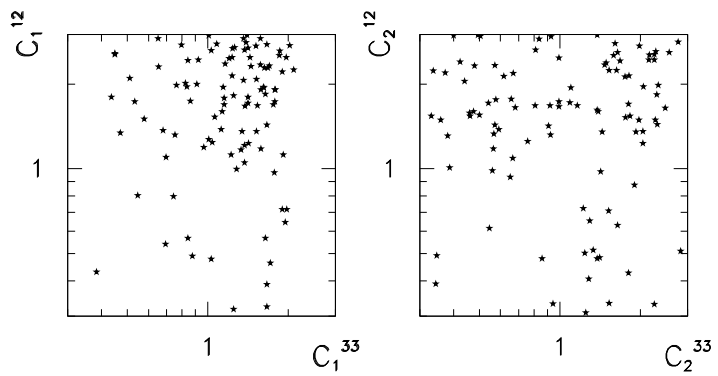

Figure 3: Scatter plots of $\left|C_{1}^{12}\right|$ versus $\left|C_{1}^{33}\right|$ (left panel) and $\left|C_{2}^{12}\right|$ versus $\left|C_{2}^{33}\right|$ (right panel) taken from 100 sets of coefficients $\left\{C_{u, 1,2}^{A B}\right\}$ reproducing the observed masses of quarks and charged leptons as well as the CKM matrix, for charge assignment $\mathbf{1}$ with $l_{A}=\bar{d}_{A}=(4,2,2)$ and $\tan \beta=15$.

Once the procedure is specified, one can generate sets of coefficients $\left\{C_{u, 1,2}^{A B}\right\}$ satisfying the above physical requirement. For illustration, we show in Fig. [3 scatter plots of the moduli of some coefficients for charge assignment $\mathbf{1}$. One does not observe any particular correlations; of course, correlations may (and presumably do) exist in more dimensional coefficient space.

The important physical features of the model are shown in Figs. [4 0 and 6 , again for charge assignment 1 with $l_{A}=\bar{d}_{A}=(4,2,2)$ and $\tan \beta=15$ taken as an example. In Fig. 团 we plot the moduli of selected elements of the rotation matrices $\mathbf{U}_{L, R}$ and $\mathbf{D}_{L, R}$ defined by

$$
U_{R}^{\dagger} \mathbf{Y}_{u} U_{L}=\operatorname{Diag}\left(y_{u}, y_{c}, y_{t}\right), \quad D_{R}^{\dagger} \mathbf{Y}_{d} D_{L}=\operatorname{Diag}\left(y_{d}, y_{s}, y_{b}\right)
$$

The phases of these matrices are chosen in such a way that the Yukawa matrix eigenvalues are real and that the CKM matrix $V=U_{L}^{\dagger} D_{L}$ is compatible with the standard PDG parametrization. We observe that $\left|U_{L}^{12}\right|$ and $\left|D_{L}^{12}\right|$ are generically both large, i.e. both the up and the down quark sectors contribute significantly to the Cabibbo angle. Similarly, for right-handed quarks, $\left|U_{R}^{12}\right|$ and $\left|D_{R}^{12}\right|$ are generically both large. Finally, the phases of the rotation matrices $\mathbf{U}_{L, R}$ and $\mathbf{D}_{L, R}$ (not shown in the plot) are random and often large.

These features are important for the discussion of FCNC processes and $\mathrm{CP}$ violating effects in supersymmetric models. Indeed, as mentioned in the introduction, the squark mass matrices depend on the rotations to the super-CKM basis, which are determined by the fermion mass models. In order to estimate the effects of these rotations on flavour and CP violating processes, we assume that, in the "flavour" basis in which the quark superfields have well-defined horizontal charges, the squark mass matrices are diagonal with non-vanishing entries, e.g.

$$
\tilde{M}_{L L}^{d 2}=\left(\begin{array}{ccc}
\tilde{m}_{1}^{2} & 0 & 0 \\
0 & \tilde{m}_{2}^{2} & 0 \\
0 & 0 & \tilde{m}_{3}^{2}
\end{array}\right) .
$$

Then, in the super-CKM basis, the off-diagonal entries of the squark mass matrix $\left.\tilde{M}_{L L}^{d 2}\right|_{S C K M}=$ 

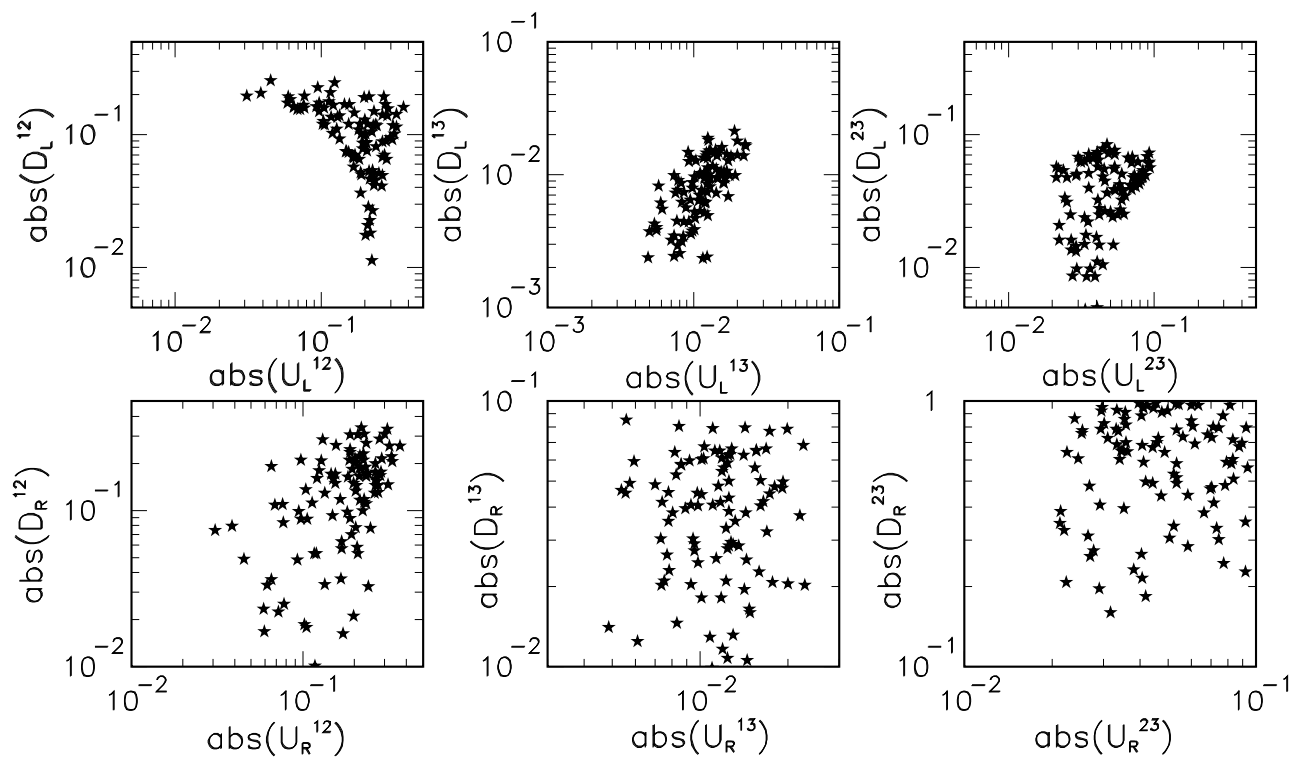

Figure 4: Scatter plots of moduli of selected entries of the rotation matrices $U_{L}, D_{L}, U_{R}$ and $D_{R}$ for charge assignment 1 with $l_{A}=\bar{d}_{A}=(4,2,2)$ and $\tan \beta=15$.

$D_{L}^{\dagger} \tilde{M}_{L L}^{d 2} D_{L}$ are given by:

$$
\begin{aligned}
\left(\tilde{M}_{L L}^{d 2}\right)^{12} & =D_{L}^{11 \star} D_{L}^{12}\left(\tilde{m}_{1}^{2}-\tilde{m}_{3}^{2}\right)+D_{L}^{21 \star} D_{L}^{22}\left(\tilde{m}_{2}^{2}-\tilde{m}_{3}^{2}\right) \\
& \simeq D_{L}^{11 \star} D_{L}^{12}\left(\tilde{m}_{1}^{2}-\tilde{m}_{2}^{2}\right) \\
\left(\tilde{M}_{L L}^{d 2}\right)^{23} & =D_{L}^{12 \star} D_{L}^{13}\left(\tilde{m}_{1}^{2}-\tilde{m}_{3}^{2}\right)+D_{L}^{22 \star} D_{L}^{23}\left(\tilde{m}_{2}^{2}-\tilde{m}_{3}^{2}\right) \\
& \simeq D_{L}^{22 \star} D_{L}^{23}\left(\tilde{m}_{2}^{2}-\tilde{m}_{3}^{2}\right) \\
\left(\tilde{M}_{L L}^{d 2}\right)^{13} & =D_{L}^{11 \star} D_{L}^{13}\left(\tilde{m}_{1}^{2}-\tilde{m}_{3}^{2}\right)+D_{L}^{21 \star} D_{L}^{23}\left(\tilde{m}_{2}^{2}-\tilde{m}_{3}^{2}\right)
\end{aligned}
$$

where we have used the fact that $D_{L}^{A B} \sim \epsilon^{\left|q_{A}-q_{B}\right|}$ to identify the dominant contribution to $\left(\tilde{M}_{L L}^{d 2}\right)^{12}$ and $\left(\tilde{M}_{L L}^{d 2}\right)^{23}$. Similar formulae hold for the off-diagonal entries of $\tilde{M}_{L L}^{u 2}$ and $\tilde{M}_{R R}^{u 2}$, with $D_{L}$ replaced by $U_{L}$ and $U_{R}$, respectively. For $\tilde{M}_{R R}^{d 2}$ one has, for charge assignments 1 and 5 :

$$
\begin{aligned}
\left(\tilde{M}_{R R}^{d 2}\right)^{12} & =D_{R}^{11 \star} D_{R}^{12}\left(\tilde{m}_{1}^{2}-\tilde{m}_{3}^{2}\right)+D_{R}^{21 \star} D_{R}^{22}\left(\tilde{m}_{2}^{2}-\tilde{m}_{3}^{2}\right) \\
\left(\tilde{M}_{R R}^{d 2}\right)^{23} & =D_{R}^{12 \star} D_{R}^{13}\left(\tilde{m}_{1}^{2}-\tilde{m}_{3}^{2}\right)+D_{R}^{22 \star} D^{23}\left(\tilde{m}_{2}^{2}-\tilde{m}_{3}^{2}\right) \\
& \simeq D_{R}^{22 \star} D_{R}^{23}\left(\tilde{m}_{2}^{2}-\tilde{m}_{3}^{2}\right) \\
\left(\tilde{M}_{R R}^{d 2}\right)^{13} & =D_{R}^{11 \star} D_{R}^{13}\left(\tilde{m}_{1}^{2}-\tilde{m}_{3}^{2}\right)+D_{R}^{21 \star} D_{R}^{23}\left(\tilde{m}_{2}^{2}-\tilde{m}_{3}^{2}\right) .
\end{aligned}
$$

Figs. [5] and [6] show the moduli and phases of the combinations of the rotation matrices $U_{L, R}$ and $D_{L, R}$ relevant for $D^{0}-\bar{D}^{0}, K^{0}-\bar{K}^{0}, B_{d}^{0}-\bar{B}_{d}^{0}$ and $B_{s}^{0}-\bar{B}_{s}^{0}$ mixing. We see that the considered hierarchical models of fermion masses may lead to large mixings in the $(1,2)$ up squark sector (the linear correlation seen in the two lower left panels of Fig. 5 is due to the symmetric form of the matrix $\mathbf{Y}_{u}$, which implies that $U_{R}=U_{L}^{\star} P$, where $P$ is a diagonal matrix of phases) and to a large mixing in the $\tilde{b}_{R}-\tilde{s}_{R}$ sector. The order of magnitude of these mixings is consistent 

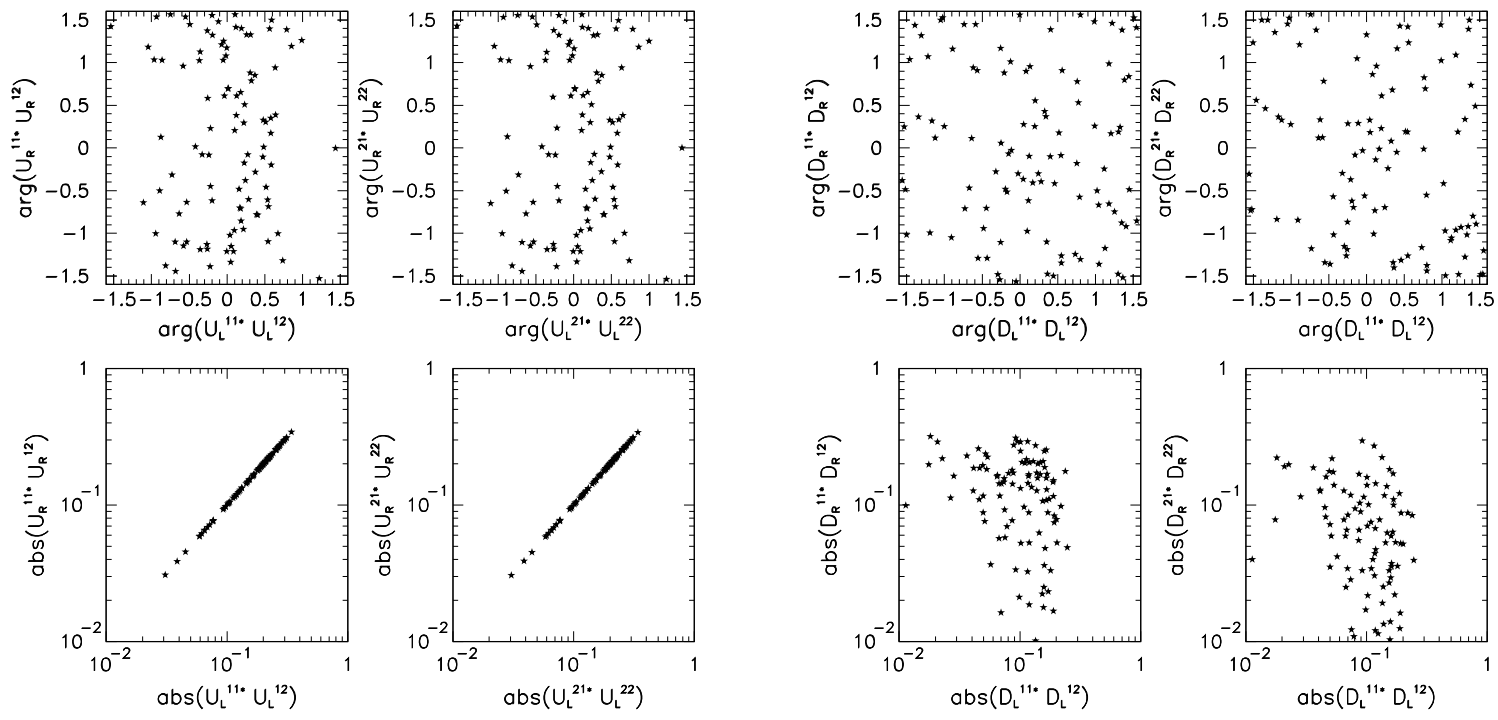

Figure 5: Scatter plots of the moduli and phases of the combinations of the rotation matrices $U_{L, R}$ and $D_{L, R}$ relevant for $D^{0}-\bar{D}^{0}$ mixing (left set of panels) and for $K^{0}-\bar{K}^{0}$ mixing (right set of panels) for charge assignment 1 with $l_{A}=\bar{d}_{A}=(4,2,2)$ and $\tan \beta=15$.
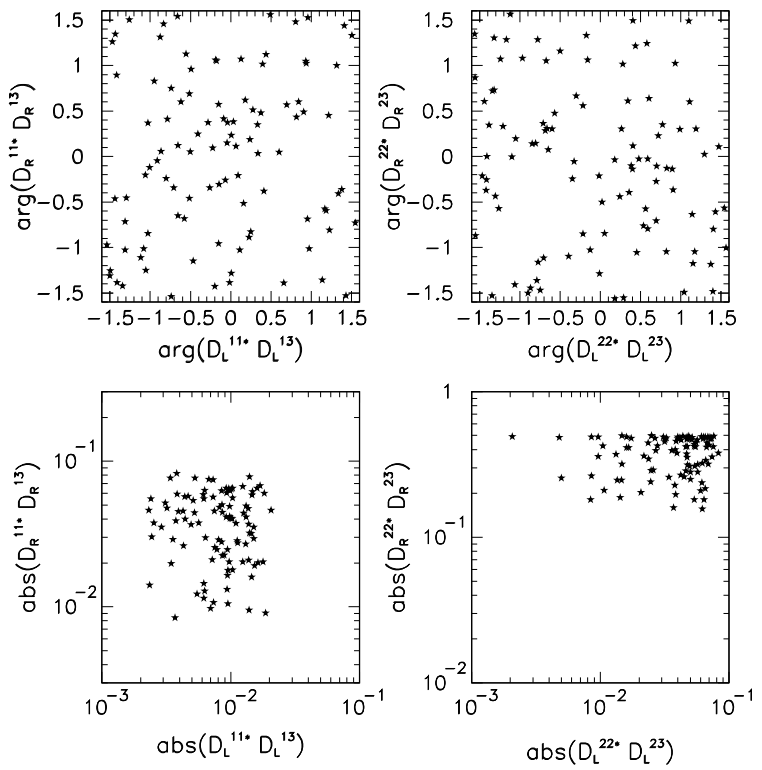

Figure 6: Scatter plots of the moduli and phases of the combinations of the rotation matrices $U_{L, R}$ and $D_{L, R}$ relevant for $B_{d^{-}}^{0} \bar{B}_{d}^{0}$ mixing (left set of panels) and for $B_{s}^{0}-\bar{B}_{s}^{0}$ mixing and $b \rightarrow s$ transitions (right set of panels) for charge assignment $\mathbf{1}$ with $l_{A}=\bar{d}_{A}=(4,2,2)$ and $\tan \beta=15$. 
with the naive "power counting": $U_{R}^{A B} \sim U_{L}^{A B} \sim D_{L}^{A B} \sim \epsilon^{\left|q_{A}-q_{B}\right|}\left(U_{R}^{A B} \sim U_{L}^{A B}\right.$ is due to the fact that $q_{A}=u_{A}$ in all phenomenologically acceptable charge assignments) and $D_{R}^{A B} \sim \epsilon^{\left|d_{A}-d_{B}\right|}$. As for the phases in the off-diagonal entries of the squark mass matrices, one can hardly see any specific pattern in the plots of Figs. 5] and 6. As one could have anticipated, these phases are essentially random.

This may lead to significant - or even excessive - contributions to flavour changing neutral currents and $\mathrm{CP}$ violating processes, especially in the kaon sector [34, 35. Significant supersymmetric contributions may also be expected in $D^{0}-\bar{D}^{0}$ mixing (with a potentially large CP-violating phase, see also Ref. [36]), as well as in $B_{s}^{0}-\bar{B}_{s}^{0}$ mixing and in $b \rightarrow s$ transitions [37. In particular, large supersymmetric contributions to CP asymmetries in e.g. $B_{d}^{0} \rightarrow \Phi K_{S}$ or $B_{s}^{0} \rightarrow J / \Psi \Phi$ are possible.

\section{Conclusions}

We have updated and put at the quantitative precision level the predictions for fermion masses and mixings in the simple Froggatt-Nielsen model based on a string-inspired gauge anomalous $U(1)$ symmetry spontaneously broken by a single flavon field, with all matter charges of the same sign. The inclusion of the neutrino oscillation data adds phenomenological constraints on the charge assignment, in addition to the Green-Schwarz anomaly cancellation conditions. Only very few charge assignments are acceptable. A precise description of fermion masses and mixings is easily obtained by adjusting order one parameters. The sets of parameters that give a good quantitative description of fermion data can be used to estimate the effects of the fermion rotations on FCNC and CP violating processes in supersymmetric models, with interesting prospects for $D^{0}-\bar{D}^{0}$ mixing, $B_{s}^{0}-\bar{B}_{s}^{0}$ mixing and $b \rightarrow s$ transitions.

\section{Acknowledgments}

This work has been supported in part by the RTN European Program MRTN-CT-2004-503369. P.H.Ch. and S.P. were supported by the Polish State Committee for Scientific Research Grant 2 P03B 12924 for 2003-2005.

\section{A Quark masses and CKM mixing angles}

In this appendix, we provide useful analytical expressions for quark mass ratios and CKM angles in models with a spontaneously broken horizontal abelian symmetry $U(1)_{X}$, at leading order in the small parameter $\epsilon \equiv\langle\phi\rangle / M$. We adopt the conventional normalization $X_{\phi}=-1$, and assume that the $X$-charges of all quark Yukawa couplings are positive, with $q_{1} \geq q_{2} \geq q_{3}$, $\bar{u}_{1} \geq \bar{u}_{2} \geq \bar{u}_{3}$ and $\bar{d}_{1} \geq \bar{d}_{2} \geq \bar{d}_{3}$. The Yukawa couplings of the up and down quarks then read:

$$
Y_{u}^{A B}=C_{u}^{A B} \epsilon^{\bar{u}_{A}+q_{B}+h_{u}}, \quad Y_{d}^{A B}=C_{d}^{A B} \epsilon^{\bar{d}_{A}+q_{B}+h_{d}},
$$

where $C_{u}^{A B}$ and $C_{d}^{A B}$ are arbitrary parameters of order one. 
We list below the expressions for the quark mass ratios $\frac{m_{u}}{m_{t}}, \frac{m_{c}}{m_{t}}, \frac{m_{d}}{m_{b}}$ and $\frac{m_{s}}{m_{b}}$, and for the CKM entries $V_{u s}, V_{c b}$ and $V_{u b}$ at leading order in the small parameter $\epsilon$. We consider the three phenomenologically relevant cases (see Section 22): (i) $\bar{d}_{1}>\bar{d}_{2}>\bar{d}_{3}$, (ii) $\bar{d}_{1}>\bar{d}_{2}=\bar{d}_{3}$ and (iii) $\bar{d}_{1}=\bar{d}_{2}>\bar{d}_{3}$, all with $q_{1}>q_{2}>q_{3}$ and $\bar{u}_{1}>\bar{u}_{2}>\bar{u}_{3}$. In order to display compact formulae, it is convenient to define the following combinations of the coefficients $C_{u}^{A B}$ :

$$
\begin{aligned}
& \Delta_{u}^{11} \equiv C_{u}^{22} C_{u}^{33}-C_{u}^{23} C_{u}^{32}, \quad \Delta_{u}^{12} \equiv C_{u}^{21} C_{u}^{33}-C_{u}^{23} C_{u}^{31}, \quad \Delta_{u}^{13} \equiv C_{u}^{21} C_{u}^{32}-C_{u}^{22} C_{u}^{31}, \\
& \Delta_{u}^{21} \equiv C_{u}^{12} C_{u}^{33}-C_{u}^{13} C_{u}^{32}, \quad \Delta_{u}^{22} \equiv C_{u}^{11} C_{u}^{33}-C_{u}^{13} C_{u}^{31}, \quad \Delta_{u}^{31} \equiv C_{u}^{12} C_{u}^{23}-C_{u}^{13} C_{u}^{22},
\end{aligned}
$$

and analogous combinations $\Delta_{d}^{A B}$ of the coefficients $C_{d}^{A B}$. Note that $\operatorname{det}\left(C_{u}^{A B}\right)=C_{u}^{11} \Delta_{u}^{11}-$ $C_{u}^{12} \Delta_{u}^{12}+C_{u}^{13} \Delta_{u}^{13}$, and similarly $\operatorname{det}\left(C_{d}^{A B}\right)=C_{d}^{11} \Delta_{d}^{11}-C_{d}^{12} \Delta_{d}^{12}+C_{d}^{13} \Delta_{d}^{13}$.

Case (i): $q_{1}>q_{2}>q_{3}, \bar{u}_{1}>\bar{u}_{2}>\bar{u}_{3}$ and $\bar{d}_{1}>\bar{d}_{2}>\bar{d}_{3}$

In this case, it is convenient to define:

$$
\bar{C}_{u}^{A B} \equiv \frac{C_{u}^{A B}}{C_{u}^{33}}, \quad \bar{C}_{d}^{A B} \equiv \frac{C_{d}^{A B}}{C_{d}^{33}} .
$$

The quark mass ratios are given by:

$$
\begin{array}{ll}
\frac{m_{u}}{m_{t}}=\left|\bar{C}_{u}^{11}-\bar{C}_{u}^{12} \frac{\Delta_{u}^{12}}{\Delta_{u}^{11}}+\bar{C}_{u}^{13} \frac{\Delta_{u}^{13}}{\Delta_{u}^{11}}\right| \epsilon^{\left(q_{1}-q_{3}\right)+\left(\bar{u}_{1}-\bar{u}_{3}\right)}, & \frac{m_{c}}{m_{t}}=\frac{\left|\Delta_{u}^{11}\right|}{\left|C_{u}^{33}\right|^{2}} \epsilon^{\left(q_{2}-q_{3}\right)+\left(\bar{u}_{2}-\bar{u}_{3}\right)}, \\
\frac{m_{d}}{m_{b}}=\left|\bar{C}_{d}^{11}-\bar{C}_{d}^{12} \frac{\Delta_{d}^{12}}{\Delta_{d}^{11}}+\bar{C}_{d}^{13} \frac{\Delta_{d}^{13}}{\Delta_{d}^{11}}\right| \epsilon^{\left(q_{1}-q_{3}\right)+\left(\bar{d}_{1}-\bar{d}_{3}\right)}, & \frac{m_{s}}{m_{b}}=\frac{\left|\Delta_{d}^{11}\right|}{\left|C_{d}^{33}\right|^{2}} \epsilon^{\left(q_{2}-q_{3}\right)+\left(\bar{d}_{2}-\bar{d}_{3}\right)},
\end{array}
$$

and the top and bottom Yukawa couplings are $y_{t}=\left|C_{u}^{33}\right| \epsilon^{q_{3}+\bar{u}_{3}}$ and $y_{b}=\left|C_{d}^{33}\right| \epsilon^{q_{3}+\bar{d}_{3}}$.

The CKM matrix entries are given by, in the standard phase convention [27]:

$$
\begin{aligned}
V_{u s} & =\left|\frac{\Delta_{d}^{12}}{\Delta_{d}^{11}}-\frac{\Delta_{u}^{12}}{\Delta_{u}^{11}}\right| \epsilon^{q_{1}-q_{2}}, \\
V_{c b} & =\left|\bar{C}_{d}^{32}-\bar{C}_{u}^{32}\right| \epsilon^{q_{2}-q_{3}}, \\
V_{u b} & =\left|\bar{C}_{d}^{31}-\bar{C}_{d}^{32} \frac{\Delta_{u}^{12}}{\Delta_{u}^{11}}+\frac{\Delta_{u}^{13}}{\Delta_{u}^{11}}\right| e^{-i \delta} \epsilon^{q_{1}-q_{3}}, \\
\delta & =\arg \left(\frac{\bar{C}_{d}^{31}-\bar{C}_{d}^{32} \frac{\Delta_{u}^{12}}{\Delta_{u}^{11}}+\frac{\Delta_{u}^{13}}{\Delta_{u}^{11}}}{\left(\frac{\Delta_{d}^{12}}{\Delta_{d}^{11}}-\frac{\Delta_{u}^{12}}{\Delta_{u}^{11}}\right)\left(\bar{C}_{d}^{32}-\bar{C}_{u}^{32}\right)}\right) .
\end{aligned}
$$

Case (ii): $q_{1}>q_{2}>q_{3}, \bar{u}_{1}>\bar{u}_{2}>\bar{u}_{3}$ and $\bar{d}_{1}>\bar{d}_{2}=\bar{d}_{3}$

In this case, it is convenient to define:

$$
\bar{C}_{u}^{A B} \equiv \frac{C_{u}^{A B}}{C_{u}^{33}}, \quad \bar{C}_{d}^{A B} \equiv \frac{C_{d}^{A B}}{\sqrt{\left|C_{d}^{23}\right|^{2}+\left|C_{d}^{33}\right|^{2}}} .
$$


The quark mass ratios are given by:

$$
\begin{gathered}
\frac{m_{u}}{m_{t}}=\left|\bar{C}_{u}^{11}-\bar{C}_{u}^{12} \frac{\Delta_{u}^{12}}{\Delta_{u}^{11}}+\bar{C}_{u}^{13} \frac{\Delta_{u}^{13}}{\Delta_{u}^{11}}\right| \epsilon^{\left(q_{1}-q_{3}\right)+\left(\bar{u}_{1}-\bar{u}_{3}\right)}, \quad \frac{m_{c}}{m_{t}}=\frac{\left|\Delta_{u}^{11}\right|}{\left|C_{u}^{33}\right|^{2}} \epsilon^{\left(q_{2}-q_{3}\right)+\left(\bar{u}_{2}-\bar{u}_{3}\right)}, \\
\frac{m_{d}}{m_{b}}=\left|\bar{C}_{d}^{11}-\bar{C}_{d}^{12} \frac{\Delta_{d}^{12}}{\Delta_{d}^{11}}+\bar{C}_{d}^{13} \frac{\Delta_{d}^{13}}{\Delta_{d}^{11}}\right| \epsilon^{\left(q_{1}-q_{3}\right)+\left(\bar{d}_{1}-\bar{d}_{3}\right)}, \quad \frac{m_{s}}{m_{b}}=\frac{\left|\Delta_{d}^{11}\right|}{\left|C_{d}^{23}\right|^{2}+\left|C_{d}^{33}\right|^{2}} \epsilon^{\left(q_{2}-q_{3}\right)+\left(\bar{d}_{2}-\bar{d}_{3}\right)},
\end{gathered}
$$

and the top and bottom Yukawa couplings are $y_{t}=\left|C_{u}^{33}\right| \epsilon^{q_{3}+\bar{u}_{3}}$ and $y_{b}=\sqrt{\left|C_{d}^{23}\right|^{2}+\left|C_{d}^{33}\right|^{2}} \epsilon^{q_{3}+\bar{d}_{3}}$.

The CKM matrix entries are given by, in the standard phase convention:

$$
\begin{aligned}
V_{u s} & =\left|\frac{\Delta_{d}^{12}}{\Delta_{d}^{11}}-\frac{\Delta_{u}^{12}}{\Delta_{u}^{11}}\right| \epsilon^{q_{1}-q_{2}}, \\
V_{c b} & =\left|\bar{C}_{d}^{22} \bar{C}_{d}^{23 \star}+\bar{C}_{d}^{32} \bar{C}_{d}^{33 \star}-\bar{C}_{u}^{32}\right| \epsilon^{q_{2}-q_{3}}, \\
V_{u b} & =\left|\left(\bar{C}_{d}^{21} \bar{C}_{d}^{23 \star}+\bar{C}_{d}^{31} \bar{C}_{d}^{33 \star}\right)-\left(\bar{C}_{d}^{22} \bar{C}_{d}^{23 \star}+\bar{C}_{d}^{32} \bar{C}_{d}^{33 \star}\right) \frac{\Delta_{u}^{12}}{\Delta_{u}^{11}}+\frac{\Delta_{u}^{13}}{\Delta_{u}^{11}}\right| e^{-i \delta} \epsilon^{q_{1}-q_{3}}, \\
\delta & =\arg \left(\frac{\left(\bar{C}_{d}^{21} \bar{C}_{d}^{23 \star}+\bar{C}_{d}^{31} \bar{C}_{d}^{33 \star}\right)-\left(\bar{C}_{d}^{22} \bar{C}_{d}^{23 \star}+\bar{C}_{d}^{32} \bar{C}_{d}^{33 \star}\right) \frac{\Delta_{u}^{12}}{\Delta_{u}^{11}}+\frac{\Delta_{u}^{13}}{\Delta_{u}^{11}}}{\left(\frac{\Delta_{d}^{12}}{\Delta_{d}^{11}}-\frac{\Delta_{u}^{12}}{\Delta_{u}^{11}}\right)\left(\bar{C}_{d}^{22} \bar{C}_{d}^{23 \star}+\bar{C}_{d}^{32} \bar{C}_{d}^{33 \star}-\bar{C}_{u}^{32}\right)}\right) .
\end{aligned}
$$

Case (iii): $q_{1}>q_{2}>q_{3}, \bar{u}_{1}>\bar{u}_{2}>\bar{u}_{3}$ and $\bar{d}_{1}=\bar{d}_{2}>\bar{d}_{3}$

In this case, it is convenient to define:

$$
\bar{C}_{u}^{A B} \equiv \frac{C_{u}^{A B}}{C_{u}^{33}}, \quad \bar{C}_{d}^{A B} \equiv \frac{C_{d}^{A B}}{C_{d}^{33}} .
$$

The quark mass ratios are given by:

$$
\begin{gathered}
\frac{m_{u}}{m_{t}}=\left|\bar{C}_{u}^{11}-\bar{C}_{u}^{12} \frac{\Delta_{u}^{12}}{\Delta_{u}^{11}}+\bar{C}_{u}^{13} \frac{\Delta_{u}^{13}}{\Delta_{u}^{11}}\right| \epsilon^{\left(q_{1}-q_{3}\right)+\left(\bar{u}_{1}-\bar{u}_{3}\right)}, \quad \frac{m_{c}}{m_{t}}=\frac{\left|\Delta_{u}^{11}\right|}{\left|C_{u}^{33}\right|^{2}} \epsilon^{\left(q_{2}-q_{3}\right)+\left(\bar{u}_{2}-\bar{u}_{3}\right)}, \\
\frac{m_{d}}{m_{b}}=\left|\bar{C}_{d}^{11} \frac{\Delta_{d}^{11}}{\sqrt{\left|\Delta_{d}^{11}\right|^{2}+\left|\Delta_{d}^{21}\right|^{2}}}-\bar{C}_{d}^{21} \frac{\Delta_{d}^{21}}{\sqrt{\left|\Delta_{d}^{11}\right|^{2}+\left|\Delta_{d}^{21}\right|^{2}}}+\bar{C}_{d}^{31} \frac{\Delta_{d}^{31}}{\sqrt{\left|\Delta_{d}^{11}\right|^{2}+\left|\Delta_{d}^{21}\right|^{2}} \mid}\right| \epsilon^{\left(q_{1}-q_{3}\right)+\left(\bar{d}_{1}-\bar{d}_{3}\right)}, \\
\frac{m_{s}}{m_{b}}=\frac{\sqrt{\left|\Delta_{d}^{11}\right|^{2}+\left|\Delta_{d}^{21}\right|^{2}}}{\left|C_{d}^{33}\right|^{2}} \epsilon^{\left(q_{2}-q_{3}\right)+\left(\bar{d}_{2}-\bar{d}_{3}\right)},
\end{gathered}
$$

and the top and bottom Yukawa couplings are $y_{t}=\left|C_{u}^{33}\right| \epsilon^{q_{3}+\bar{u}_{3}}$ and $y_{b}=\left|C_{d}^{33}\right| \epsilon^{q_{3}+\bar{d}_{3}}$.

The CKM matrix entries are given by, in the standard phase convention:

$$
\begin{aligned}
V_{u s} & =\left|\frac{\Delta_{d}^{12} \Delta_{d}^{11 \star}+\Delta_{d}^{22} \Delta_{d}^{21 \star}}{\left|\Delta_{d}^{11}\right|^{2}+\left|\Delta_{d}^{21}\right|^{2}}-\frac{\Delta_{u}^{12}}{\Delta_{u}^{11}}\right| \epsilon^{q_{1}-q_{2}}, \\
V_{c b} & =\left|\bar{C}_{d}^{32}-\bar{C}_{u}^{32}\right| \epsilon^{q_{2}-q_{3}},
\end{aligned}
$$




$$
\begin{aligned}
V_{u b} & =\left|\bar{C}_{d}^{31}-\bar{C}_{d}^{32} \frac{\Delta_{u}^{12}}{\Delta_{u}^{11}}+\frac{\Delta_{u}^{13}}{\Delta_{u}^{11}}\right| e^{-i \delta} \epsilon^{q_{1}-q_{3}}, \\
\delta & =\arg \left(\frac{\bar{C}_{d}^{31}-\bar{C}_{d}^{32} \frac{\Delta_{u}^{12}}{\Delta_{u}^{11}}+\frac{\Delta_{u}^{13}}{\Delta_{u}^{11}}}{\left(\frac{\Delta_{d}^{12} \Delta_{d}^{11 \star}+\Delta_{d}^{22} \Delta_{d}^{21 \star}}{\left|\Delta_{d}^{11}\right|^{2}+\left|\Delta_{d}^{21}\right|^{2}}-\frac{\Delta_{u}^{12}}{\Delta_{u}^{11}}\right)\left(\bar{C}_{d}^{32}-\bar{C}_{u}^{32}\right)}\right) .
\end{aligned}
$$

\section{B Lepton masses and PMNS mixing angles}

In this appendix, we provide analytical expressions for lepton masses and PMNS angles in models with a spontaneously broken horizontal abelian symmetry $U(1)_{X}$, at leading order in the small parameter $\epsilon \equiv\langle\phi\rangle / M$. We adopt the conventional normalization $X_{\theta}=-1$, and assume that the $X$-charges of all lepton Yukawa couplings and right-handed neutrino mass terms are positive, with $l_{1} \geq l_{2} \geq l_{3}, \bar{n}_{1} \geq \bar{n}_{2} \geq \bar{n}_{3}$ and $\bar{e}_{1} \geq \bar{e}_{2} \geq \bar{e}_{3}$. The Yukawa couplings of the charged leptons and the Dirac and Majorana matrices of the neutrinos then read:

$$
Y_{e}^{A B}=C_{e}^{A B} \epsilon^{\bar{e}_{A}+l_{B}+h_{d}}, \quad Y_{D}^{A B}=C_{D}^{A B} \epsilon^{\bar{n}_{A}+l_{B}+h_{u}}, \quad M_{M}^{A B}=M_{R} C_{M}^{A B} \epsilon^{\bar{n}_{A}+\bar{n}_{B}}
$$

where $M_{R}$ is the scale of right-handed neutrino masses, and $C_{D}^{A B}, C_{e}^{A B}$ and $C_{M}^{A B}=C_{M}^{B A}$ are arbitrary parameters of order one. The seesaw mechism leads to the effective light neutrino mass matrix:

$$
M_{\nu}^{A B}=C_{\nu}^{A B} \frac{v_{u}^{2}}{M_{R}} \epsilon^{l_{A}+l_{B}+2 h_{u}}, \quad C_{\nu}^{A B}=C_{\nu}^{B A}=-\sum_{C, D} C_{D}^{C A} C_{D}^{D B}\left(C_{M}^{-1}\right)^{C D}
$$

where we have used the fact that $\left(M_{M}^{-1}\right)^{C D}=M_{R}^{-1}\left(C_{M}^{-1}\right)^{C D} \epsilon^{-\bar{n}_{C}-\bar{n}_{D}}$. It is a well-known fact that $M_{\nu}$ does not depend on the hierarchy of right-handed neutrinos (i.e. on the $\bar{n}_{A}$ ) when $\bar{n}_{A}+l_{B}+h_{u} \geq 0$ and $\bar{n}_{A}+\bar{n}_{B} \geq 0$, but only on the order one coefficients $C_{M}^{A B}$. It is then legitimate, from the low-energy point of view, to consider the $C_{\nu}^{A B}$ as arbitrary order one coefficients. As in the quark sector, we define combinations $\Delta_{e}^{A B}$ and $\Delta_{\nu}^{A B}$ of the order one coefficients $C_{e}^{A B}$ and $C_{\nu}^{A B}$ in order to display compact formulae for the lepton masses and mixings. For simplicity and given the fact that $C P$ violation has not been observed in the lepton sector yet, we shall assume that the coefficients $C_{e}^{A B}$ and $C_{\nu}^{A B}$ are real.

Given the structure (80), neutrino data are best accommodated by $l_{2}=l_{3}$ and $l_{1}>l_{3}$, with the additional requirement that $x \equiv \frac{\left|\Delta_{\nu}^{11}\right|}{\left(C_{\nu}^{22}+C_{\nu}^{33}\right)^{2}} \ll 1$, to be explained by a (mild) cancellation among order one coefficients in $\Delta_{\nu}^{11}$, in order to account for the hierarchy between the solar and the atmospheric neutrino mass scales. In practice, only the cases $\epsilon^{n} \ll x \ll 1$ and $\epsilon^{n} \sim x$, where $n \equiv l_{1}-l_{3}=1$ or 2 , can accommodate the LMA solution, and we shall discuss each of them in turn. Since the neutrino mass spectrum is hierarchical, the right-handed neutrino mass scale has to be adjusted to $M_{R} \sim v_{u}^{2} \epsilon^{2\left(l_{3}+h_{u}\right)} / \sqrt{\Delta m_{\text {atm }}^{2}} \simeq\left(6 \times 10^{14} \mathrm{GeV}\right) \epsilon^{2\left(l_{3}+h_{u}\right)}$. The mass hierarchy in the charged lepton sector requires $\bar{e}_{1}>\bar{e}_{2}>\bar{e}_{3}$ for $\epsilon \sim \lambda$, given that the acceptable values for $n$ are 1 and 2 , as we shall see below. 
The charged lepton mass ratios are given by:

$$
\frac{m_{e}}{m_{\tau}}=\left|\bar{C}_{e}^{11}-\bar{C}_{e}^{12} \frac{\Delta_{e}^{12}}{\Delta_{e}^{11}}+\bar{C}_{e}^{13} \frac{\Delta_{e}^{13}}{\Delta_{e}^{11}}\right| \epsilon^{\left(l_{1}-l_{3}\right)+\left(\bar{e}_{1}-\bar{e}_{3}\right)}, \quad \frac{m_{\mu}}{m_{\tau}}=\frac{\left|\Delta_{e}^{11}\right|}{\left(C_{e}^{32}\right)^{2}+\left(C_{e}^{33}\right)^{2}} \epsilon^{\left(\bar{e}_{2}-\bar{e}_{3}\right)}
$$

where $\bar{C}_{e}^{A B} \equiv C_{e}^{A B} / \sqrt{\left(C_{e}^{32}\right)^{2}+\left(C_{e}^{33}\right)^{2}}$, and the tau Yukawa coupling is $y_{\tau}=\sqrt{\left(C_{e}^{32}\right)^{2}+\left(C_{e}^{33}\right)^{2}} \epsilon^{l_{3}+\bar{e}_{3}+h_{d}}$. The atmospheric and "CHOOZ" mixing angles $\theta_{23}$ and $\theta_{13}$ are given by:

$$
\begin{aligned}
\tan \theta_{23} & =\left|\frac{C_{e}^{33} C_{\nu}^{22}+C_{e}^{32} C_{\nu}^{23}}{C_{e}^{32} C_{\nu}^{22}-C_{e}^{33} C_{\nu}^{23}}\right| \\
\sin \theta_{13} & =\left|\frac{\left(\Delta_{e}^{12} C_{\nu}^{22}+\Delta_{e}^{13} C_{\nu}^{23}\right)\left(C_{\nu}^{22}+C_{\nu}^{33}\right)+\Delta_{e}^{11}\left(C_{\nu}^{12} C_{\nu}^{22}+C_{\nu}^{13} C_{\nu}^{23}\right)}{\Delta_{e}^{11}\left(C_{\nu}^{22}+C_{\nu}^{33}\right) \sqrt{\left(C_{\nu}^{23}\right)^{2}+\left(C_{\nu}^{33}\right)^{2}}}\right| \epsilon^{n} .
\end{aligned}
$$

One naturally ends up with a large atmospheric mixing angle ( $\tan \theta_{23} \sim 1$ ), as a result of the choice $l_{2}=l_{3}$, and with $\sin \theta_{13} \sim \epsilon^{n}$, which can be very close to the present experimental upper limit for $n=1$.

As for the neutrino masses and the solar mixing angle $\theta_{12}$, their formulae depend on whether $\epsilon^{n} \ll x$ or $\epsilon^{n} \sim x$.

Case 1: $\epsilon^{n} \ll x \ll 1$. The neutrino masses are given by, in units of $\frac{v_{u}^{2}}{M_{R}}$ and at leading order in $x$ and $\epsilon^{n}$ :

$$
\begin{aligned}
m_{\nu_{1}} & =\frac{\left|C_{\nu}^{11} \Delta_{\nu}^{11}-C_{\nu}^{12} \Delta_{\nu}^{12}+C_{\nu}^{13} \Delta_{\nu}^{13}\right|}{\left(C_{\nu}^{22}+C_{\nu}^{33}\right)^{2}} \frac{\epsilon^{2 n}}{x} \\
m_{\nu_{2}} & =\left|C_{\nu}^{22}+C_{\nu}^{33}\right| x \\
m_{\nu_{3}} & =\left|C_{\nu}^{22}+C_{\nu}^{33}\right|(1-x)
\end{aligned}
$$

The ratio of solar to atmospheric neutrino oscillation frequencies is then approximately given by:

$$
\frac{\Delta m_{21}^{2}}{\Delta m_{32}^{2}} \simeq x^{2}
$$

and the solar mixing angle reads:

$$
\tan \theta_{12}=\left|\frac{\Delta_{\nu}^{12}}{\left(C_{\nu}^{22}+C_{\nu}^{33}\right) \sqrt{\left(C_{\nu}^{23}\right)^{2}+\left(C_{\nu}^{33}\right)^{2}}}\right| \frac{\epsilon^{n}}{x} .
$$

Neutrino oscillation data tell us that $\frac{\Delta m_{21}^{2}}{\Delta m_{32}^{2}} \approx 0.2$, hence $x \approx 0.2$. For $n=2$ this is consistent with the present experimentally allowed range for $\tan \theta_{12}\left(0.28 \leq \tan ^{2} \theta_{12} \leq 0.58\right.$ at $3 \sigma$ C.L. [38] $)$ provided that the order one coefficients conspire to give $\Delta_{\nu}^{12} /\left(C_{\nu}^{22}+C_{\nu}^{33}\right) \sqrt{\left(C_{\nu}^{23}\right)^{2}+\left(C_{\nu}^{33}\right)^{2}} \sim(2-3)$. The "CHOOZ" angle in this scheme is $\sin \theta_{13} \sim \epsilon^{2} \simeq 0.05$, which corresponds to $\sin ^{2} 2 \theta_{13} \sim 0.01$, a value that should be accessible to the coming neutrino superbeam experiments $\mathrm{T} 2 \mathrm{~K}$ and $\mathrm{NO} \nu \mathrm{A}$. 
Case 2: $\epsilon^{n} \sim x \ll 1$. The neutrino masses are given by, in units of $\frac{v_{u}^{2}}{M_{R}}$ and at leading order in $\epsilon^{n} \sim x$ :

$$
\begin{aligned}
& m_{\nu_{1}}=\left|C_{\nu}^{22}+C_{\nu}^{33}\right|\left|1-\sqrt{1-\frac{4 \operatorname{det} N}{\left(C_{\nu}^{22}+C_{\nu}^{33}\right)^{3}} \frac{\epsilon^{2 n}}{x^{2}}}\right| \frac{x}{2}, \\
& m_{\nu_{2}}=\left|C_{\nu}^{22}+C_{\nu}^{33}\right|\left(1+\sqrt{1-\frac{4 \operatorname{det} N}{\left(C_{\nu}^{22}+C_{\nu}^{33}\right)^{3}} \frac{\epsilon^{2 n}}{x^{2}}}\right) \frac{x}{2}, \\
& m_{\nu_{3}}=\left|C_{\nu}^{22}+C_{\nu}^{33}\right| .
\end{aligned}
$$

The ratio of solar to atmospheric neutrino oscillation frequencies then reads:

$$
\frac{\Delta m_{21}^{2}}{\Delta m_{32}^{2}}=x^{2} \sqrt{1-\frac{4 \operatorname{det} N}{\left(C_{\nu}^{22}+C_{\nu}^{33}\right)^{3}} \frac{\epsilon^{2 n}}{x^{2}}} \sim x^{2},
$$

and the solar mixing angle is given by:

$$
\tan ^{2} \theta_{12}=\frac{\left(\Delta_{\nu}^{12} \Delta_{\nu}^{13}\right)^{2}+\left(C_{\nu}^{23}\right)^{2}\left[\left(\Delta_{\nu}^{12}\right)^{2}+\left(\Delta_{\nu}^{13}\right)^{2}\right]\left(m_{\nu_{1}} / \epsilon^{n}\right)^{2}}{\left(\Delta_{\nu}^{12} \Delta_{\nu}^{13}\right)^{2}+\left(C_{\nu}^{23}\right)^{2}\left[\left(\Delta_{\nu}^{12}\right)^{2}+\left(\Delta_{\nu}^{13}\right)^{2}\right]\left(m_{\nu_{2}} / \epsilon^{n}\right)^{2}}
$$

Neutrino oscillation data require $x \sim 0.2$, hence $n=1$. The solar mixing angle naturally falls into the experimentally allowed range, and the "CHOOZ" angle is predicted to be close to its present upper limit, $\sin \theta_{13} \sim \epsilon \simeq 0.22$, which could be tested already by the MINOS, CNGS (OPERA and ICARUS) or D-CHOOZ experiments.

\section{References}

[1] C.D. Frogatt and H.B. Nielsen, Nucl. Phys. B147 (1979) 277.

[2] J. Bijnens and C. Wetterich, Nucl. Phys. B283 (1987) 237, Phys. Lett. B199 (1987) 525.

[3] M. Leurer, Y. Nir and N. Seiberg, Nucl. Phys. B398 (1993) 319, Nucl. Phys. B420 (1994) 468.

[4] L.E. Ibanez and G.G. Ross, Phys. Lett. B332 (1994) 100.

[5] P. Binetruy and P. Ramond, Phys. Lett. B350 (1995) 49.

[6] V. Jain and R. Shrock, Phys. Lett. B352 (1995) 83.

[7] E. Dudas, S. Pokorski and C.A. Savoy, Phys. Lett. B356 (1995) 45.

[8] Y. Nir, Phys. Lett. B354 (1995) 107.

[9] P. Binetruy, S. Lavignac and P. Ramond, Nucl. Phys. B477 (1996) 353. 
[10] Z. Berezhiani and Z. Tavartkiladze, Phys. Lett. B 396 (1997) 150; K. Choi, E.J. Chun and H.D. Kim, Phys. Lett. B394 (1997) 89; N. Irges, S. Lavignac and P. Ramond, Phys. Rev. D58 (1998) 035003; Y. Grossman, Y. Nir and Y. Shadmi, JHEP 9810 (1998) 007; C.D. Froggatt, M. Gibson and H.B. Nielsen, Phys. Lett. B446 (1999) 256; Q. Shafi and Z. Tavartkiladze, Phys. Lett. B 451 (1999) 129; G.K. Leontaris and J. Rizos, Nucl. Phys. B567 (2000) 32; J.R. Ellis, G.K. Leontaris and J. Rizos, JHEP 0005 (2000) 001; A.S. Joshipura, R.D. Vaidya and S.K. Vempati, Phys. Rev. D62 (2000) 093020; N. Maekawa, Prog. Theor. Phys. 106 (2001) 401; M. Kakizaki and M. Yamaguchi, JHEP 0206 (2002) 032; K.S. Babu, I. Gogoladze and K. Wang, Nucl. Phys. B660 (2003) 322; K.S. Babu, T. Enkhbat and I. Gogoladze, Nucl. Phys. B678 (2004) 233; I. Jack, D.R.T. Jones and R. Wild, Phys. Lett. B580 (2004) 72; H.K. Dreiner, H. Murayama and M. Thormeier, arXiv:hep-ph/0312012.

[11] D.B. Kaplan and M. Schmaltz, Phys. Rev. D49 (1994) 3741; A. Pomarol and D. Tommasini, Nucl. Phys. B466 (1996) 3; L.J. Hall and H. Murayama, Phys. Rev. Lett. 75 (1995) 3985; R. Barbieri, G.R. Dvali and L.J. Hall, Phys. Lett. B377 (1996) 76; C.D. Carone, L.J. Hall and H. Murayama, Phys. Rev. D54 (1996) 2328; R. Barbieri, L.J. Hall, S. Raby and A. Romanino, Nucl. Phys. B493 (1997) 3; R. Dermisek and S. Raby, Phys. Rev. D62 (2000) 015007; T. Blazek, S. Raby and K. Tobe, Phys. Rev. D62 (2000) 055001; Z. Berezhiani and A. Rossi, Nucl. Phys. B594 (2001) 113; R.G. Roberts, A. Romanino, G.G. Ross and L. Velasco-Sevilla, Nucl. Phys. B615 (2001) 358; S.F. King and G.G. Ross, Phys. Lett. B520 (2001) 243, Phys. Lett. B574 (2003) 239; M.C. Chen and K.T. Mahanthappa, Int. J. Mod. Phys. A18 (2003) 5819.

[12] M.B. Green and J.H. Schwarz, Phys. Lett. 149B (1984) 117.

[13] P. Minkowski, Phys. Lett. B67 (1977) 421; M. Gell-Mann, P. Ramond, and R. Slansky, Talk given at the 19th Sanibel Symposium, Palm Coast, Florida, Feb. 25-Mar. 2, 1979, preprint CALT-68-709 (retro-print hep-ph/9809459), and in Supergravity, North Holland, Amsterdam, 1980, p. 315; T. Yanagida, in Proc. of the Workshop on Unified Theories and Baryon Number in the Universe, Tsukuba, Japan, Feb. 13-14, 1979, p 95; S. Glashow, in Quarks and Leptons, Cargèse Lectures, July 9-29 1979, Plenum, New York, 1980, p. 687; R.N. Mohapatra and G. Senjanović, Phys. Rev. Lett. 44 (1980) 912.

[14] N. Irges, S. Lavignac and P. Ramond, Phys. Rev. D58 (1998) 035003.

[15] A. Sagnotti, Phys. Lett. B294 (1992) 196.

[16] L.E. Ibanez, Phys. Lett. B303 (1993) 55.

[17] G.-F. Giudice and A. Masiero, Phys. Lett. B206 (1988) 480.

[18] M. Dine, N. Seiberg and E. Witten, Nucl. Phys. B289 (1987) 589.

[19] J.J. Atick, L.J. Dixon and A. Sen, Nucl. Phys. B292 (1987) 109; M. Dine, I. Ichinose and N. Seiberg, Nucl. Phys. B293 (1987) 253. 
[20] A.E. Nelson and D. Wright, Phys. Rev. D56 (1997) 1598.

[21] S. Dimopoulos and G.-F. Giudice, Phys. Lett. B357 (1995) 573.

[22] N. Irges and S. Lavignac, Phys. Lett. B424 (1998) 293.

[23] M. Olechowski and S. Pokorski, Phys. Lett. B257 (1991) 388.

[24] K. S. Babu, Z. Phys. C35 (1987) 69.

[25] V. Barger, M.S. Berger and P. Ohmann, Phys. Rev. D47 (1993) 1093, 2038.

[26] P. H. Chankowski and S. Pokorski, Int. J. Mod. Phys. A17 (2002) 575.

[27] Review of Particle Properties, S. Eidelman et al., Phys. Lett. B592 (2004) 1.

[28] P. H. Chankowski, W. Królikowski and S. Pokorski, Phys. Lett. B473 (2000) 109; J.A. Casas, J.R. Espinosa, A. Ibarra and I. Navarro, Nucl. Phys. B573 (2000) 652.

[29] G. Altarelli and F. Feruglio, Phys. Rept. 320 (1999) 295; G. Altarelli and F. Feruglio, in Neutrino Mass, Springer Tracts in Modern Physics, ed. by G. Altarelli and K. Winter, preprint CERN-TH-2002-127 (arXiv:hep-ph/0206077).

[30] G. Altarelli, F. Feruglio and I. Masina JHEP 0301 (2003) 035 (updated in v2 of hep-ph/0210342).

[31] H. Georgi and C. Jarlskog, Phys. Lett. B86 (1979) 297.

[32] J. Ellis and M.K. Gaillard, Phys. Lett. B88 (1979) 315.

[33] G. Altarelli, F. Feruglio and I. Masina JHEP 0011 (2000) 040.

[34] F. Gabbiani, E. Gabrielli, A. Masiero and L. Silvestrini, Nucl. Phys. B477 (1996) 321.

[35] M. Ciuchini et al., JHEP 9810 (1998) 008.

[36] Y. Nir and G. Raz, Phys. Rev. D66 (2002) 035007.

[37] M. Ciuchini, E. Franco, A. Masiero and L. Silvestrini, Phys. Rev. D67 (2003) 075016 [Erratum-ibid. D68 (2003) 079901].

[38] J.N. Bahcall, M.C. Gonzalez-Garcia and C. Pena-Garay, JHEP 0408 (2004) 016. 